\title{
MAXIMAL REGULAR BOUNDARY VALUE PROBLEMS IN BANACH-VALUED WEIGHTED SPACE
}

\author{
RAVI P. AGARWAL, MARTIN BOHNER, AND VELI B. SHAKHMUROV
}

Received 10 July 2004

This study focuses on nonlocal boundary value problems for elliptic ordinary and partial differential-operator equations of arbitrary order, defined in Banach-valued function spaces. The region considered here has a varying bound and depends on a certain parameter. Several conditions are obtained that guarantee the maximal regularity and Fredholmness, estimates for the resolvent, and the completeness of the root elements of differential operators generated by the corresponding boundary value problems in Banachvalued weighted $L_{p}$ spaces. These results are applied to nonlocal boundary value problems for regular elliptic partial differential equations and systems of anisotropic partial differential equations on cylindrical domain to obtain the algebraic conditions that guarantee the same properties.

\section{Introduction and notation}

Boundary value problems for differential-operator equations have been studied in detail in $[4,15,22,35,40,42]$. The solvability and the spectrum of boundary value problems for elliptic differential-operator equations have also been studied in $[5,6,12,14,16,18,29$, $30,31,32,33,34,37,41]$. A comprehensive introduction to differential-operator equations and historical references may be found in [22, 42]. In these works, Hilbert-valued function spaces have been considered. The main objective of the present paper is to discuss nonlocal boundary value problems for ordinary and partial differential-operator equations (DOE) in Banach-valued weighted $L_{p}$ spaces. In this work, the following is done.

(1) The continuity, compactness, and qualitative properties of the embedding operators in the associated Banach-valued weighted function space are considered.

(2) An ordinary differential-operator equation

$$
L u=\sum_{k=0}^{m} a_{k} A_{\lambda}^{m-k} u^{(k)}(x)=f(x), \quad x \in(0, b), a_{m} \neq 0
$$

of arbitrary order on a domain with varying bound is investigated. 
(3) An anisotropic partial DOE

$$
\sum_{k=1}^{n} a_{k} D_{k}^{l_{k}} u(x)+\sum_{|\alpha: l|<1} A_{\alpha}(x) D^{\alpha} u(x)=f(x), \quad x=\left(x_{1}, x_{2}, \ldots, x_{n}\right)
$$

is investigated.

(4) Both boundary conditions are, in general, nonlocal.

(5) The operators in equations and boundary conditions are, in general, unbounded. Note that certain classes of degenerate equations of the above types are considered in $L_{p}$ spaces, after transforming them with suitable substitutions to equations in weighted $L_{p}$ spaces.

In the present work, we address the maximal regularity, Fredholmness, qualitative properties of the resolvent, and the completeness of the root elements of differential operators that are generated by these boundary value problems. These results are applied to nonlocal boundary value problems for elliptic and quasielliptic partial differential equations with parameters and their finite or infinite systems on cylindrical domains. In Section 1, some notation and definitions are given. In Section 2, certain background material concerning embedding theorems between Banach-valued weighted function spaces is presented. These spaces consist of functions that belong to $E_{0}$-valued weighted $L_{p}$ space and their generalized anisotropic derivatives with respect to different variables belonging to $E$-valued weighted $L_{p}$ space. In this section, we show that there exist some mixed derivatives of these functions that belong to $\left(E_{0}, E\right)_{\theta}$-valued weighted $L_{p}$ spaces, where $\left(E_{0}, E\right)_{\theta}$ are interpolation spaces between $E_{0}$ and $E$, and the parameter $\theta$ depends on the order of mixed differentiations and the order of spaces. Embedding theorems of such type have been investigated in [24] for Hilbert-valued $L_{2}$ spaces. In Section 3, coercive estimates in terms of the interpolation spaces $\left(E\left(A^{m}\right), E\right)_{\theta}$ of the nonlocal boundary value problems for the underlying homogeneous ordinary DOE are proved. Next, in Section 4, we show that the boundary value problem for the above ordinary DOE generates an isomorphism (algebraically and topologically) between corresponding $E\left(A^{m}\right)$ valued Sobolev spaces and

$$
L_{p}(0, b ; E) \times \prod_{k=1}^{n}\left(E\left(A^{m}\right), E\right)_{\theta_{k}}
$$

where $\theta_{k}$ depends on $m$ and on the order of the boundary conditions. In Section 5, the maximal regularity and Fredholmness of nonlocal boundary value problems on a cylindrical domain for the above underlying anisotropic partial DOE are investigated. In Section 6, estimates for the resolvent and the completeness of the root elements of differential operators generated by these boundary value problems are shown. Finally, in Section 7, the maximal regularity and Fredholmness of nonlocal boundary value problems for anisotropic partial differential equations and for their infinite systems, in general, are proved.

Let $\gamma=\gamma(x)$ be a positive measurable weight function on the region $\Omega \subset \mathbb{R}^{n}$. Let $L_{p, \gamma}(\Omega ; E)$ denote the space of strongly measurable $E$-valued functions that are defined 
on $\Omega$ with the norm

$$
\|f\|_{p, \gamma}=\|f\|_{L_{p, \gamma}(\Omega ; E)}=\left(\int\|f(x)\|_{E}^{p} \gamma(x) d x\right)^{1 / p}, \quad 1 \leq p<\infty .
$$

For $\gamma(x) \equiv 1$, the space $L_{p, \gamma}(\Omega ; E)$ will be denoted by $L_{p}(\Omega ; E)$ with norm $\|f\|_{p}$. By $L_{\mathbf{p}, \gamma}(\Omega)$ and $W_{\mathbf{p}, \gamma}^{l}(\Omega), \mathbf{p}=\left(p_{1}, p_{2}\right)$ we will denote a $p$-summable weighted function space and weighted Sobolev space (see $[7,38]$ ) with mixed norm, respectively.

The Banach space $E$ is said to be $\xi$-convex $[8,9]$ if there exists on $E \times E$ a symmetric real-valued function $\xi$ which is convex with respect to each of the variables and satisfies the conditions

$$
\xi(0,0)>0, \quad \xi(u, v) \leq\|u+v\| \quad \text { for }\|u\|=\|v\|=1 .
$$

The $\xi$-convex Banach space $E$ is often called a UMD space and written as $E \in$ UMD. It is shown in [9] that a Hilbert operator $(H f)(x)=\lim _{\varepsilon \rightarrow 0} \int_{|y|>\varepsilon} f(y) /(x-y) d y$ is bounded in $L_{p}(R, E), p \in(1, \infty)$, for those and only those spaces $E$ which satisfy $E \in \mathrm{UMD}$. UMD spaces include, for example, $L_{p}, l_{p}$ spaces, and Lorentz spaces $L_{p q}$ with $p, q \in(1, \infty)$.

Let $\mathbb{C}$ be the set of complex numbers and

$$
S_{\varphi}=\{\lambda \in \mathbb{C}:|\arg \lambda-\pi| \leq \pi-\varphi\} \cup\{0\}, \quad 0<\varphi \leq \pi .
$$

A linear operator $A$ is said to be $\varphi$-positive in a Banach space $E$ with bound $M>0$ if $D(A)$ is dense in $E$ and

$$
\left\|(A-\lambda I)^{-1}\right\|_{L(E)} \leq M(1+|\lambda|)^{-1}
$$

with $\lambda \in S_{\varphi}, \varphi \in(0, \pi]$, where $I$ is the identity operator in $E$ and $L(E)$ is the space of bounded linear operators acting on $E$. Sometimes, instead of $A+\lambda I$ we will write $A+\lambda$ and denote this by $A_{\lambda}$. It is known [38, Section 1.15.1] that there exist fractional powers $A^{\theta}$ of the positive operator $A$. Let $E\left(A^{\theta}\right)$ denote the space $D\left(A^{\theta}\right)$ with graphical norm defined as

$$
\|u\|_{E\left(A^{\theta}\right)}=\left(\|u\|^{p}+\left\|A^{\theta} u\right\|^{p}\right)^{1 / p}, \quad 1 \leq p<\infty,-\infty<\theta<\infty .
$$

Let $E_{0}$ and $E$ be two Banach spaces and let $E_{0}$ be continuously and densely embedded into $E$. By $\left(E_{0}, E\right)_{\theta, p}, 0<\theta<1,1 \leq p \leq \infty$, we will denote interpolation spaces for $\left\{E_{0}, E\right\}$ by the $K$ method [38, Section 1.3.1].

Let $l$ be an integer and $(a, b) \subset \mathbb{R}=(-\infty, \infty)$. Let $W_{p, \gamma}^{l}(a, b ; E)$ denote the $E$-valued weighted Sobolev space of the functions $u \in L_{p, \gamma}(a, b ; E)$ that have generalized derivatives $u^{(k)}(x) \in L_{p, \gamma}(a, b ; E)$ included on $(a, b)$ up to the $l$ th order and with the norm

$$
\|u\|_{W_{p, \gamma}^{l}(a, b ; E)}=\sum_{k=0}^{l}\left(\int_{a}^{b}\left\|u^{(k)}(x)\right\|_{E}^{p} \gamma(x) d x\right)^{1 / p}<\infty .
$$

Consider the Banach space

$$
W_{p, \gamma}^{l}\left(a, b ; E_{0}, E\right)=L_{p, \gamma}\left(a, b ; E_{0}\right) \cap W_{p, \gamma}^{l}(a, b ; E)
$$


12 Maximal regular BVPs in Banach-valued weighted space

with the norm

$$
\|u\|_{W_{p, \gamma}^{l}\left(a, b ; E_{0}, E\right)}=\|u\|_{L_{p, \gamma}\left(a, b ; E_{0}\right)}+\left\|u^{(l)}\right\|_{L_{p, \gamma}(a, b ; E)}<\infty
$$

Let $E_{1}$ and $E_{2}$ be two Banach spaces. A function

$$
\Psi \in C\left(\mathbb{R}^{n} ; L\left(E_{1}, E_{2}\right)\right)
$$

is called a multiplier from $L_{p, \gamma}\left(\mathbb{R}^{n} ; E_{1}\right)$ to $L_{q, \gamma}\left(\mathbb{R}^{n} ; E_{2}\right)$ if there exists a constant $C>0$ with

$$
\left\|F^{-1} \Psi(\xi) F u\right\|_{L_{q, \gamma}\left(\mathbb{R}^{n} ; E_{2}\right)} \leq C\|u\|_{L_{p, \gamma}\left(\mathbb{R}^{n} ; E_{1}\right)}
$$

for all $u \in L_{p, \gamma}\left(\mathbb{R}^{n} ; E_{1}\right)$, where $F$ is the Fourier transformation. The set of all multipliers from $L_{p, \gamma}\left(\mathbb{R}^{n} ; E_{1}\right)$ to $L_{q, \gamma}\left(\mathbb{R}^{n} ; E_{2}\right)$ will be denoted by $M_{p, \gamma}^{q, \gamma}\left(E_{1}, E_{2}\right)$. For $E_{1}=E_{2}=E$, it will be denoted by $M_{p, \gamma}^{q, \gamma}(E)$. Let

$$
H_{k}=\left\{\Psi_{h} \in M_{p, \gamma}^{q, \gamma}\left(E_{1}, E_{2}\right): h=\left(h_{1}, h_{2}, \ldots, h_{n}\right) \in K\right\}
$$

be a collection of multipliers in $M_{p, \gamma}^{q, \gamma}\left(E_{1}, E_{2}\right)$. We say that $H_{k}$ is a uniform collection of multipliers if there exists a constant $M_{0}>0$, independent of $h \in K$, with

$$
\left\|F^{-1} \Psi_{h} F u\right\|_{L_{p, \gamma}\left(\mathbb{R}^{n} ; E_{2}\right)} \leq M_{0}\|u\|_{L_{p, \gamma}\left(\mathbb{R}^{n} ; E_{1}\right)}
$$

for all $h \in K$ and $u \in L_{p, \gamma}\left(\mathbb{R}^{n} ; E_{1}\right)$. The theory of multipliers of the Fourier transformation and some related references can be found in [38, Section 2.2.1] (for vector-valued functions see, e.g., $[26,28])$.

A set $K \subset B\left(E_{1}, E_{2}\right)$ is called $R$-bounded (see $[8,39]$ ) if there exists a constant $C>0$ such that for all $T_{1}, T_{2}, \ldots, T_{m} \in K$ and $u_{1}, u_{2}, \ldots, u_{m} \in E_{1}, m \in \mathbb{N}$,

$$
\int_{0}^{1}\left\|\sum_{j=1}^{m} r_{j}(y) T_{j} u_{j}\right\|\left\|_{E_{2}} d y \leq C \int_{0}^{1}\right\| \sum_{j=1}^{m} r_{j}(y) u_{j} \|_{E_{1}} d y,
$$

where $\left\{r_{j}\right\}$ is a sequence of independent symmetric $[-1,1]$-valued random variables on $[0,1]$. Now, let

$$
\begin{aligned}
& V_{n}=\left\{\left(\xi_{1}, \xi_{2}, \ldots, \xi_{n}\right) \in \mathbb{R}^{n}: \xi_{j} \neq 0\right\}, \\
& U_{n}=\left\{\beta=\left(\beta_{1}, \beta_{2}, \ldots, \beta_{n}\right):|\beta| \leq n\right\} .
\end{aligned}
$$

Definition 1.1. A Banach space $E$ is said to be a space satisfying a multiplier condition with respect to $p \in(1, \infty)$ and weight function $\gamma$ if the following condition holds: if $\Psi \in$ $C^{(n)}\left(\mathbb{R}^{n} ; B(E)\right)$ and the set

$$
\left\{\xi^{\beta} D_{\xi}^{\beta} \Psi(\xi): \xi \in V_{n}, \beta \in U_{n}\right\}
$$

is $R$-bounded, then $\Psi \in M_{p, \gamma}^{p, \gamma}(E)$. 
Definition 1.2. The positive operator $A$ is said to be $R$-positive in the Banach space $E$ if there exists $\varphi \in(0, \pi]$ such that the set

$$
L_{A}=\left\{(1+|\xi|)(A-\xi I)^{-1}: \xi \in S_{\varphi}\right\}
$$

is $R$-bounded.

Note that in Hilbert spaces every norm bounded set is $R$-bounded. Therefore, in Hilbert spaces all positive operators are $R$-positive. If $A$ is a generator of a contraction semigroup on $L_{q}, 1 \leq q \leq \infty$ [25], then $A$ has bounded imaginary powers with $\left\|\left(-A^{i t}\right)\right\|_{B(E)} \leq C e^{\nu|t|}, v<\pi / 2$ [11] or if $A$ is a generator of a semigroup with Gaussian bound [16] in $E \in \mathrm{UMD}$, then those operators are $R$-positive.

It is well known (see, e.g., [25]) that any Hilbert space satisfies the multiplier condition. By virtue of [28], Mikhlin conditions are not sufficient for the operator-valued multiplier theorem. There are, however, Banach spaces which are not Hilbert spaces but satisfy the multiplier condition, for example, UMD spaces (see $[8,9,39]$ ).

A linear operator $A(t)$ is said to be uniformly $\varphi$-positive with respect to $t$ in $E$ if $D(A(t))$ is independent of $t, D(A(t))$ is dense in $E$, and

$$
\left\|(A(t)-\lambda I)^{-1}\right\| \leq \frac{M}{1+|\lambda|}
$$

for all $\lambda \in S(\varphi)$, where $\varphi \in(0, \pi]$.

For two sequences $\left\{a_{j}\right\}_{j \in \mathbb{N}}$ and $\left\{b_{j}\right\}_{j \in \mathbb{N}}$ of positive numbers, the expression $a_{j} \sim b_{j}$ means that there exist positive numbers $C_{1}$ and $C_{2}$ such that

$$
C_{1} a_{j} \leq b_{j} \leq C_{2} a_{j} \quad \forall j \in \mathbb{N} \text {. }
$$

Let $\sigma_{\infty}\left(E_{1}, E_{2}\right)$ denote the space of compact operators acting from $E_{1}$ to $E_{2}$. For $E_{1}=$ $E_{2}=E$, this space will be denoted by $\sigma_{\infty}(E)$. Denote by $s_{j}(I)$ and $d_{j}(I)$ the approximation numbers and $d$-numbers of the operator $I$, respectively, (see, e.g., [38, Section 1.16.1]). Let

$$
\sigma_{q}\left(E_{1}, E_{2}\right)=\left\{A \in \sigma_{\infty}\left(E_{1}, E_{2}\right): \sum_{j=1}^{\infty} s_{j}^{q}(A)<\infty, 1 \leq q<\infty\right\} .
$$

Let $\Omega \subset \mathbb{R}^{n}$ and $l=\left(l_{1}, l_{2}, \ldots, l_{n}\right)$. Suppose $\beta_{k}=\beta_{k}(x)$ are positive measurable functions on $\Omega$. We consider the Banach-valued function space $W_{p, \beta, \gamma}^{l}\left(\Omega ; E_{0}, E\right)$ which consists of the functions $u \in L_{p, \gamma}\left(\Omega ; E_{0}\right)$ that have the generalized derivatives $D_{k}^{l_{k}} u=\partial^{l_{k}} u / \partial x_{k}^{l_{k}}$ such that $\beta_{k} D_{k}^{l_{k}} u \in L_{p, \gamma}(\Omega ; E), k \in\{1,2, \ldots, n\}$ with the norm

$$
\|u\|_{W_{p, \beta, \gamma}^{l}\left(\Omega ; E_{0}, E\right)}=\|u\|_{L_{p, \gamma}\left(\Omega ; E_{0}\right)}+\sum_{k=1}^{n}\left\|\beta_{k} D_{k}^{l_{k}} u\right\|_{L_{p, \gamma}(\Omega ; E)}<\infty .
$$

For $\beta_{k}(x) \equiv 1, k \in\{1,2, \ldots, n\}$, the space $W_{p, \beta, \gamma}^{l}\left(\Omega ; E_{0}, E\right)$ will be denoted by $W_{p, \gamma}^{l}\left(\Omega ; E_{0}, E\right)$. For $\gamma(x) \equiv 1$, the space $W_{p, \gamma}^{l}\left(\Omega ; E_{0}, E\right)$ will be denoted by $W_{p}^{l}\left(\Omega ; E_{0}, E\right)$. For $E_{0}=E$, this 
14 Maximal regular BVPs in Banach-valued weighted space

space is denoted by $W_{p}^{l}(\Omega ; E)$. Let $t=\left(t_{1}, t_{2}, \ldots, t_{n}\right)$, where $t_{j}>0$ are parameters. We define in $W_{p, \gamma}^{l}\left(\Omega ; E_{0}, E\right)$ the parameter norm

$$
\|u\|_{W_{p, \gamma, t}^{l}\left(\Omega ; E_{0}, E\right)}=\|u\|_{L_{p, \gamma}\left(\Omega ; E_{0}\right)}+\sum_{k=1}^{n}\left\|t_{k} D_{k}^{l_{k}} u\right\|_{L_{p, \gamma}(\Omega ; E)} .
$$

The weights $\gamma$ are said to satisfy an $A_{p}$ condition, that is, $\gamma \in A_{p}$ with $1<p<\infty$, if there exists a constant $C$ such that

$$
\left(\frac{1}{|Q|} \int_{Q} \gamma(x) d x\right)\left(\frac{1}{|Q|} \int_{Q} \gamma^{-1 /(p-1)}(x) d x\right)^{p-1} \leq C
$$

for all cubes $Q \subset \mathbb{R}^{n}$.

\section{Embedding theorems}

Let $\alpha=\left(\alpha_{1}, \alpha_{2}, \ldots, \alpha_{n}\right)$ and $D^{\alpha}=D_{1}^{\alpha_{1}} D_{2}^{\alpha_{2}} \cdots D_{n}^{\alpha_{n}}$. Using a similar technique as in $[29,32$, 33], we obtain the following result.

THEOREM 2.1. Let the following conditions be satisfied:

(1) $\gamma=\gamma(x)$ is a weight function satisfying the $A_{p}$ condition;

(2) $E$ is a Banach space satisfying the multiplier condition with respect to $p$ and weight function $\gamma$;

(3) $A$ is an R-positive operator in $E$ and $t=\left(t_{1}, t_{2}, \ldots, t_{n}\right), 0<t_{k}<t_{0}<\infty$;

(4) $\alpha=\left(\alpha_{1}, \alpha_{2}, \ldots, \alpha_{n}\right)$ and $l=\left(l_{1}, l_{2}, \ldots, l_{n}\right)$ are $n$-tuples of nonnegative integer numbers such that

$$
\varkappa=\left|\left(\alpha+\frac{1}{p}-\frac{1}{q}\right): l\right|=\sum_{k=1}^{n} \frac{\alpha_{k}+1 / p}{l_{k}} \leq 1, \quad 1<p<\infty, 0 \leq \mu \leq 1-\varkappa ;
$$

(5) $\Omega \subset \mathbb{R}^{n}$ is a region such that there exists a bounded linear extension operator acting from $L_{p, \gamma}(\Omega ; E)$ to $L_{p, \gamma}\left(\mathbb{R}^{n} ; E\right)$ and also from $W_{p, \gamma}^{l}(\Omega ; E(A), E)$ to $W_{p, \gamma}^{l}\left(\mathbb{R}^{n} ; E(A), E\right)$.

Then, an embedding

$$
D^{\alpha} W_{p, \gamma}^{l}(\Omega ; E(A), E) \subset L_{p, \gamma}\left(\Omega ; E\left(A^{1-\varkappa-\mu}\right)\right)
$$

is continuous and there exists a positive constant $C_{\mu}$ such that

$$
\prod_{k=1}^{n} t_{k}^{\alpha_{k} / l_{k}}\left\|D^{\alpha} u\right\|_{L_{p, \gamma}\left(\Omega ; E\left(A^{1-\varkappa-\mu}\right)\right)} \leq C_{\mu}\left[h^{\mu}\|u\|_{W_{p, \gamma, t}^{l}(\Omega ; E(A), E)}+h^{-(1-\mu)}\|u\|_{L_{p, \gamma}(\Omega ; E)}\right]
$$

for all $u \in W_{p, \gamma}^{l}(\Omega ; E(A), E)$ and $0<h \leq h_{0}<\infty$. 
Proof. It suffices to prove the estimate (2.3). In fact, first, the estimate (2.3) is proved for $\Omega=\mathbb{R}^{n}$. The estimate (2.3) for $\Omega=\mathbb{R}^{n}$ will follow if we prove the inequality

$$
\begin{aligned}
& \prod_{k=1}^{n} t_{k}^{\alpha_{k} / l_{k}}\left\|F^{-1}\left[(i \xi)^{\alpha} A^{1-\varkappa-\mu} \hat{u}\right]\right\|_{L_{p, \gamma}\left(\mathbb{R}^{n}, E\right)} \\
& \quad \leq C_{\mu}\left\|F^{-1}\left[h^{\mu}\left(A+\sum_{k=1}^{n} t_{k}\left(\delta\left(\xi_{k}\right) \xi_{k}\right)^{l_{k}}\right)+h^{-(1-\mu)}\right] \hat{u}\right\|_{L_{p, \gamma}\left(\mathbb{R}^{n}, E\right)},
\end{aligned}
$$

where $\delta \in C^{\infty}(\mathbb{R})$ with $\delta(y) \geq 0$ for all $y \geq 0, \delta(y)=0$ for $|y| \leq 1 / 2, \delta(-y)=-\delta(y)$ for all $y$, and

$$
\xi^{\alpha}=\xi_{1}^{\alpha_{1}} \xi_{2}^{\alpha_{2}} \ldots \xi_{n}^{\alpha_{n}}
$$

It is clear that (2.4) will follow if we can prove that the operator-function

$$
\Psi_{t}(\xi)=\prod_{k=1}^{n} t_{k}^{\alpha_{k} / l_{k}} \xi^{\alpha} A^{1-\varkappa-\mu} h^{-\mu}\left[A+\sum_{k=1}^{n} t_{k}\left(\delta\left(\xi_{k}\right)\right)^{l_{k}}+h^{-1}\right]^{-1}
$$

is a multiplier in $L_{p, \gamma}\left(\mathbb{R}^{n} ; E\right)$, which is uniform with respect to the parameters $t$ and $h$. Then, by using the moment inequality for powers of positive operators and the Young inequality as in $[32,33,34]$ we obtain

$$
\left\|\Psi_{t}(\xi) u\right\|_{E} \leq C\|Q(\xi) u\|_{E}+\|A Q(\xi) u\|_{E}
$$

where

$$
Q(\xi)=\left[A+\sum_{k=1}^{n} t_{k}\left(\delta\left(\xi_{k}\right)\right)^{l_{k}}+h^{-1}\right]^{-1}
$$

Thus, in view of (2.7), due to $R$-positivity of the operator $A$ (or, applying [39, Lemma 3.8], we can obtain this for UMD spaces), we find that the function $\Psi_{t}$ is a multiplier in $L_{p, \gamma}(\mathbb{R} ; E)$. Therefore, we obtain the estimate $(2.4)$. Then, by using the extension operator in $W_{p, \gamma}^{l}(\Omega ; E(A), E)$, from $(2.4)$ we obtain $(2.3)$.

By applying a similar technique as in $[29,31]$ we obtain the following.

Theorem 2.2. Suppose conditions (1)-(3) of Theorem 2.1 are satisfied. Suppose $\Omega$ is a bounded region in $\mathbb{R}^{n}$ and an embedding $E_{0} \subset E$ is compact. Then, an embedding

$$
W_{p, \gamma}^{l}(\Omega ; E(A), E) \subset L_{p, \gamma}(\Omega ; E)
$$

is compact.

Theorem 2.3. Suppose all conditions of Theorem 2.1 are satisfied and suppose $\Omega$ is a bounded region in $\mathbb{R}^{n}, A^{-1} \in \sigma_{\infty}(E)$. Then, for $0<\mu \leq 1-\varkappa$ an embedding (2.2) is compact. 
16 Maximal regular BVPs in Banach-valued weighted space

Proof. Putting in (2.3) $h=\|u\|_{L_{p, y}(\Omega ; E)} /\|u\|_{W_{p, \gamma}^{l}(\Omega ; E(A), E)}$, we obtain a multiplicative inequality

$$
\left\|D^{\alpha} u\right\|_{L_{p, \gamma}\left(\Omega ; E\left(A^{1-\varkappa-\mu}\right)\right)} \leq C_{\mu}\|u\|_{L_{p, \gamma}(\Omega ; E)}^{\mu}\|u\|_{W_{p, \gamma}^{l}(\Omega ; E(A), E)^{\circ}}^{1-\mu}
$$

By virtue of Theorem 2.2 the embedding $W_{p \gamma}^{l}(\Omega ; E(A), E) \subset L_{p, \gamma}(\Omega ; E)$ is compact. Then, from the estimate (2.10) we obtain the assertion.

Similarly as in Theorem 2.1 we obtain the following result.

Theorem 2.4. Suppose all conditions of Theorem 2.1 are satisfied. Then, for $0<\mu<1-\varkappa$ an embedding

$$
D^{\alpha} W_{p, \gamma}^{l}(\Omega ; E(A), E) \subset L_{p, \gamma}\left(\Omega ;(E(A), E)_{\varkappa, p}\right)
$$

is continuous and there exists a positive constant $C_{\mu}$ such that

$$
\begin{aligned}
& \prod_{k=1}^{n} t_{k}^{\alpha_{k} / l_{k}}\left\|D^{\alpha} u\right\|_{L_{p, \gamma}\left(\Omega ;(E(A), E)_{\varkappa+\mu, p}\right)} \\
& \quad \leq C_{\mu}\left[h^{\mu}\left(\|A u\|_{L_{p, \gamma}(\Omega ; E)}+\sum_{k=1}^{n}\left\|t_{k} D_{k}^{l_{k}} u\right\|_{L_{p, \gamma}(\Omega ; E)}\right)+h^{-(1-\mu)}\|u\|_{L_{p, \gamma}(\Omega ; E)}\right]
\end{aligned}
$$

for all $u \in W_{p, \gamma}^{l}(\Omega ; E(A), E)$ and $0<h \leq h_{0}<\infty$.

Similarly as in Theorem 2.2 the following result can be shown.

Theorem 2.5. Suppose all conditions of Theorem 2.2 are satisfied. Then for $0<\mu<1-\varkappa$ an embedding (2.11) is compact.

Theorem 2.6 [34]. Let $E$ be a Banach space, $A$ a $\varphi$-positive operator in $E$ with bound $M$, $\varphi \in(0, \pi / 2)$. Let $m, l \in \mathbb{N}, 1 \leq p<\infty$, and $\alpha \in(1 / 2 p, m+1 / 2 p), 0 \leq v<2 p \alpha-1$. Then, for $\lambda \in S(\varphi)$ an operator, $-A_{\lambda}^{1 / l}$ generates a semigroup $e^{-A_{\lambda}^{1 / l} x}$ which is holomorphic for $x>0$. Moreover, there exists a constant $C>0$ (depending only on $M, \varphi, m, \alpha$, and $p$ ) such that for every $u \in\left(E, E\left(A^{m}\right)\right)_{\alpha l / 2 m-(1+v) / 2 m p, p}$ and $\lambda \in S(\varphi)$,

$$
\begin{aligned}
\int_{0}^{\infty} \| & (A+\lambda I)^{\alpha} e^{-x(A+\lambda I)^{1 / l}} u \|^{p} x^{\nu} d x \\
& \leq C\left[\|u\|_{\left(E, E\left(A^{m}\right)\right)_{\alpha / / 2 m-(1+\nu) / 2 m p, p}^{p}}^{p}+|\lambda|^{\alpha l p / 2-(1+\nu) / 2}\|u\|_{E}^{p}\right] .
\end{aligned}
$$

Proof. By using a similar technique as in [12, Lemma 2.2], at first for a $\varphi$-positive operator $A$, where $\varphi \in(\pi / 2, \pi)$, and for every $u \in E$ such that

$$
\int_{0}^{\infty}\left\|x^{\alpha-(1+\nu) / p}\left(A(A+x)^{-1}\right)^{m} u\right\|^{p} x^{\nu-1} d x<\infty,
$$


using the integral representation formula for holomorphic semigroups, we obtain an estimate

$$
\int_{0}^{\infty}\left\|A^{\alpha} e^{-x A} u\right\|^{p} x^{\nu} d x \leq C \int_{0}^{\infty}\left\|x^{\alpha-(1+\nu) / p}\left(A(A+x)^{-1}\right)^{m} u\right\|^{p} x^{\nu-1} d x .
$$

Then, by using the above estimate and [12, Lemmas 2.3-2.5] we obtain the assertion.

Let $\bar{\Omega}$ denote the closure of the region $\Omega$. Similarly as in [7, Theorem 10.4] we obtain the following.

THeorem 2.7. Suppose the following conditions are satisfied:

(1) $\gamma=\gamma(x)$ is a weight function satisfying the $A_{p}$ condition;

(2) $E$ is a Banach space and $\alpha=\left(\alpha_{1}, \alpha_{2}, \ldots, \alpha_{n}\right), l=\left(l_{1}, l_{2}, \ldots, l_{n}\right), 1 \leq p \leq \infty, \varkappa=$ $\sum_{k=1}^{n}\left(\alpha_{k}+1 / p\right) / l_{k}<1$

(3) $\Omega \subset \mathbb{R}^{n}$ is a region satisfying the l-horn condition [7, page 117].

Then, the embedding $D^{\alpha} W_{p, \gamma}^{l}(\Omega ; E) \subset C(\bar{\Omega} ; E)$ holds, and there exists a constant $M>0$ such that

$$
\left\|D^{\alpha} u\right\|_{C(\bar{\Omega} ; E)} \leq M\left[h^{1-\varkappa}\|u\|_{W_{p, \gamma}^{l}(\Omega ; E)}+h^{-\varkappa}\|u\|_{L_{p, \gamma}(\Omega ; E)}\right]
$$

for all $u \in W_{p, y}^{l}(\Omega ; E)$ and $0<h \leq h_{0}<\infty$.

Let

$$
G=\left\{x=\left(x_{1}, x_{2}, \ldots, x_{n}\right): 0<x_{k}<T_{k}\right\}, \quad \gamma(x)=x_{1}^{\gamma_{1}} x_{2}^{\gamma_{2}} \cdots x_{n}^{\gamma_{n}} .
$$

Let $\beta_{k}=x_{k}^{\beta_{k}}, \nu=\prod_{k=1}^{n} x_{k}^{\nu_{k}}, \gamma=\prod_{k=1}^{n} x_{k}^{\gamma_{k}}$. Let $I=I\left(W_{p, \beta, \gamma}^{l}(\Omega ; E(A), E), L_{p, \gamma}(\Omega ; E)\right)$ be the embedding operator

$$
W_{p, \beta, \gamma}^{l}(\Omega ; E(A), E) \longrightarrow L_{p, \nu}(\Omega ; E)
$$

Using a similar technique as in [30] and [38, Section 3.8], we obtain the following result. Theorem 2.8. Suppose that $E$ is a Banach space with base and

$$
\begin{array}{cc}
0 \leq \gamma_{k}<p-1, & 0 \leq \beta_{k}<1, \quad \nu_{k}-\gamma_{k}>p\left(\beta_{k}-1\right), \\
1<p<\infty, & s_{j}\left(I\left(E_{0}, E\right)\right) \sim j^{-1 / k_{0}}, \quad k_{0}>0, j \in \mathbb{N}, \\
\varkappa_{0}=\sum_{k=1}^{n} \frac{\gamma_{k}-\nu_{k}}{p\left(l_{k}-\beta_{k}\right)}<1 .
\end{array}
$$

Then,

$$
s_{j}\left(I\left(W_{p, \beta, \gamma}^{l}\left(G ; E_{0}, E\right), L_{p, \nu}(G ; E)\right)\right) \sim j^{-1 /\left(k_{0}+\varkappa_{0}\right)} .
$$


18 Maximal regular BVPs in Banach-valued weighted space

Proof. By the partial polynomial approximation method (see, e.g., [38, Section 3.8]), we obtain that there exist positive constants $C_{1}$ and $C_{2}$ such that

$$
\begin{aligned}
& s_{j}\left(I\left(W_{p, \beta, \gamma}^{l}\left(G ; E_{0}, E\right), L_{p, \nu}(G ; E)\right)\right) \leq C_{1} j^{-1 /\left(k_{0}+\varkappa_{0}\right)}, \\
& d_{j}\left(I\left(W_{p, \beta, \gamma}^{l}\left(G ; E_{0}, E\right), L_{p, \nu}(G ; E)\right)\right) \geq C_{2} j^{-1 /\left(k_{0}+\varkappa_{0}\right)} .
\end{aligned}
$$

Therefore, from the above estimates and by virtue of the inequality $d_{j}(I) \leq s_{j}(I)$ (see $[38$, Section 1.16.1]), we obtain the assertion.

Consider a principal differential-operator equation

$$
L u=u^{(m)}(x)+\sum_{k=1}^{m} a_{k} A^{k} u^{(m-k)}(x)+(B u)(x)=0, \quad x \in(0, b) .
$$

Let $\omega_{1}, \omega_{2}, \ldots, \omega_{m}$ be the roots of the equation

$$
\omega^{m}+a_{1} \omega^{m-1}+\cdots+a_{m}=0
$$

and let

$$
\begin{aligned}
& \omega_{m}=\min \left\{\arg \omega_{j}, j=1, \ldots, v ; \arg \omega_{j}+\pi, j=v+1, \ldots, m\right\}, \\
& \omega_{M}=\max \left\{\arg \omega_{j}, j=1, \ldots, v ; \arg \omega_{j}+\pi, j=v+1, \ldots, m\right\} .
\end{aligned}
$$

A system of numbers $\omega_{1}, \omega_{2}, \ldots, \omega_{m}$ is called $\nu$-separated if there exists a straight line $P$ passing through 0 such that no value of the numbers $\omega_{j}$ lies on it, and $\omega_{1}, \omega_{2}, \ldots, \omega_{\nu}$ are on one side of $P$, while $\omega_{\nu+1}, \ldots, \omega_{m}$ are on the other.

As in [42, Lemma 5.3.2/1], we obtain the following result.

LEMMA 2.9. Let the following conditions be satisfied:

(1) $a_{m} \neq 0$ and the roots of (2.23), $\omega_{j}, j=1, \ldots, m$, are $v$-separated;

(2) $A$ is a closed operator in the Banach space $E$ with a dense domain $D(A)$ and

$$
\left\|(A-\lambda I)^{-1}\right\| \leq C|\lambda|^{-1}, \quad-\frac{\pi}{2}-\omega_{M} \leq \arg \lambda \leq \frac{\pi}{2}-\omega_{m}, \quad|\lambda| \longrightarrow \infty .
$$

Then, for a function $u(x)$ to be a solution of (2.22), which belongs to the space $W_{p}^{m}(0, b$; $\left.E\left(A^{m}\right), E\right)$, it is necessary and sufficient that

$$
u(x)=\sum_{k=1}^{v} e^{-x \omega_{k} A} g_{k}+\sum_{k=v+1}^{m} e^{-(b-x) \omega_{k} A} g_{k}
$$

where

$$
g_{k} \in\left(E\left(A^{m}\right), E\right)_{1 / m p, p}, \quad k=1,2, \ldots, m
$$


Statement of the problems. Let $\Omega$ be a region in $\mathbb{R}^{N}$ and $\bar{\Omega}$ the closure of $\Omega$. Let $t=$ $\left(t_{1}, t_{2}, \ldots, t_{N}\right) \in \Omega$ and let $b(t)$ be positive continuous functions on the region $\bar{\Omega}$. Consider a nonlocal boundary value problem

$$
\begin{gathered}
L u:=\sum_{k=0}^{m} a_{k} A_{\lambda}^{m-k} u^{(k)}(x)=f(x), \quad x \in(0, b), a_{m} \neq 0, \\
L_{k} u:=\sum_{i=0}^{v_{k}}\left[\alpha_{k i} u^{(i)}(0)+\beta_{k i} u^{(i)}(b(t))+\sum_{j=1}^{N_{k}} \delta_{k i j} u^{(i)}\left(x_{k j t}\right)\right]=f_{k}, \quad k=1,2, \ldots, m,
\end{gathered}
$$

in a Banach space $E$, on the varying region $0 \leq x \leq b(t)$, where $0 \leq v_{k} \leq m-1$ and $\alpha_{k i}$, $\beta_{k i}, \delta_{k i j}$ are complex-valued functions depending on the domain parameters $t$ and $x_{k j t} \in$ $(0, b(t))$ for $t \in \Omega$. Moreover, $A_{\lambda}=A+\lambda, A$, and $T_{k j}$ are, generally speaking, unbounded operators in $E$. We denote $\alpha_{k v_{k}}, \beta_{k v_{k}}$, and $\delta_{k j v_{k}}$ by $\alpha_{k}, \beta_{k}$, and $\delta_{k j}$, respectively.

Let $\gamma(x)=x^{\gamma}$. The functions belonging to the space $W_{p}^{m}\left(0, b ; E\left(A^{m}\right), E\right)$ and satisfying $L u=f(x)$ a.e. on $(0, b)$ are called solutions of $(2.28)$ on $(0, b)$. Let

$$
G=\left\{(x, y) \in \mathbb{R}^{2}: 0<x<1,0<y<h(x)\right\},
$$

where $h$ is a continuous function on $[0,1]$. We now consider a boundary value problem

$$
\begin{aligned}
& L_{0} u:= a_{1} D_{x}^{l_{1}} u(x, y)+a_{2} D_{y}^{l_{2}} u(x, y)+A_{\lambda} u(x, y) \\
&+\sum_{|\alpha: l|<1} A_{\alpha}(x, y) D^{\alpha} u(x, y)=f(x, y), \\
& L_{1 j} u=0, \quad j=1,2, \ldots, l_{1}, \quad L_{2 j} u=0, \quad j=1,2, \ldots, l_{2},
\end{aligned}
$$

where

$$
\begin{aligned}
L_{1 j} u:= & \sum_{i=0}^{m_{1 j}}\left[\alpha_{1 j i} u_{x}^{(i)}(0, y)+\beta_{1 j i} u_{x}^{(i)}(1, y)+\sum_{\nu=1}^{N_{1 j}} \delta_{1 j i v} u_{x}^{(i)}\left(x_{j v}, y\right)\right] \\
& +\sum_{\nu=1}^{M_{1 j}} T_{1 j \nu} u\left(x_{\nu 0}, y\right)=0, \quad j=1,2, \ldots, l_{1}, \\
L_{2 j} u:= & \sum_{i=0}^{m_{2 j}}\left[\alpha_{2 j i} u_{y}^{(i)}(x, 0)+\beta_{2 j i} u_{y}^{(i)}(x, h)+\sum_{\nu=1}^{N_{2 j}} \delta_{2 j i v} u_{y}^{(i)}\left(x, \Gamma_{j \nu}\right)\right] \\
& +\sum_{\nu=1}^{M_{2 j}} T_{2 j \nu} u\left(x, \Gamma_{\nu 0}\right)=0, \quad j=1,2, \ldots, l_{2},
\end{aligned}
$$

in $E$, where $A_{\lambda}=A+\lambda, A, A_{\alpha}(x, y)$ and $T_{k j v}$ are, generally speaking, unbounded operators in $E, 0<x_{\nu}<1,0 \leq x_{\nu 0} \leq 1,0<\Gamma_{\nu}<h(x), 0 \leq \Gamma_{\nu 0} \leq h(x)$, and $a_{k}, \alpha_{k j i}, \beta_{j i}, \delta_{k j i v}$ are complex numbers,

$$
0 \leq m_{k j} \leq l_{k}-1 .
$$


We denote $\alpha_{k j m_{k j}}$ by $\alpha_{k j}$ and $\beta_{k j m_{k j}}$ by $\beta_{k j}, k=1,2$. Let

$$
\begin{gathered}
D_{1}^{\alpha_{1}}=D_{x}^{\alpha_{1}}=\frac{\partial^{\alpha_{1}}}{\partial x^{\alpha_{1}}}, \quad D_{2}^{\alpha_{2}}=D_{y}^{\alpha_{2}}=\frac{\partial^{\alpha_{2}}}{\partial y^{\alpha_{2}}}, \quad D^{\alpha}=D_{x}^{\alpha_{1}} D_{y}^{\alpha_{2}} \\
\gamma_{1}(x)=x^{\gamma_{1}}, \quad \gamma_{2}(y)=y^{\gamma_{2}}, \quad \gamma=\gamma(x, y)=x^{\gamma_{1}} y^{\gamma_{2}}
\end{gathered}
$$

Functions belonging to the space $W_{p, \gamma}^{l}(G ; E(A), E), l=\left(l_{1}, l_{2}\right)$, satisfying (2.31) a.e. on $G$ and boundary conditions (2.32) are called solutions of the boundary value problem (2.31), (2.32) on $G$.

\section{Homogeneous equations}

Let $L$ be defined as in (2.28) and consider the homogeneous equation

$$
L u=0
$$

together with the boundary conditions $(2.29)$. Let $\omega_{1}, \omega_{2}, \ldots, \omega_{m}$ be the roots of the characteristic equation

$$
a_{m} \omega^{m}+a_{1} \omega^{m-1}+\cdots+a_{0}=0 .
$$

Let $\left[v_{n k}\right]_{n, k=1,2, \ldots, m}$ be an $m \times m$-matrix with determinant $\omega(t)=\operatorname{det}\left[v_{n k}\right]$, where

$$
v_{n k}= \begin{cases}\alpha_{k}(t)\left(-\omega_{n}\right)^{m_{k}} & \text { for } n=1,2, \ldots, d, k=1,2, \ldots, m, \\ \beta_{k}(t) \omega_{n}^{m_{k}} & \text { for } n=d+1, d+2, \ldots, m, k=1,2, \ldots, m .\end{cases}
$$

Theorem 3.1. Let the following conditions be satisfied:

(1) $A$ is a $\varphi$-positive operator in a Banach space $E$ for $\varphi \in(0, \pi / 2)$;

(2) $\omega(t)=\operatorname{det}\left[v_{n k}\right] \neq 0$ for all $t \in \Omega$, and $\eta_{k}=\left(p m+p v_{k}+1\right) / 2 p m, k=1,2, \ldots, m$;

(3) $a_{m} \neq 0$ and $\left|\arg \omega_{j}-\pi\right| \leq \pi / 2-\varphi, j=1,2, \ldots, d,\left|\arg \omega_{j}\right| \leq \pi / 2-\varphi, j=d+1, \ldots$, $m, 0<d<m$;

(4) $\alpha_{k i}, \beta_{k i}, \delta_{k j i}$ are continuous functions on $\bar{\Omega}$.

Then, the problem (3.1), (2.29) for

$$
f_{k} \in E_{k}=\left(E\left(A^{m}\right), E\right)_{\eta_{k}, p}, \quad p \in(1, \infty), \quad|\arg \lambda| \leq \pi-\varphi
$$

and sufficiently large $|\lambda|$ has a unique solution $u \in W_{p}^{m}\left(0, b ; E\left(A^{m}\right), E\right)$, and coercive uniformity with respect to $t$ and $\lambda$, that is,

$$
\sum_{k, j=0}^{m}(1+|\lambda|)^{m-k-j}\left\|A^{k} u^{(j)}\right\|_{L_{p}(0, b ; E)} \leq M \sum_{k=1}^{m}\left(\left\|f_{k}\right\|_{E_{k}}+|\lambda|^{1-\theta_{k}}\left\|f_{k}\right\|_{E}\right)
$$

holds for the solution of (3.1), (2.29), where $k+j \leq m$.

Proof. From condition (1), by virtue of Theorem 2.6, for $|\arg \lambda| \leq \pi-\varphi$, there exists the semigroup $e^{-A_{\lambda} x}$, and it is holomorphic for $x>0$ and strongly continuous for $x \geq 0$. By 
virtue of Lemma 2.9, an arbitrary solution of (3.1) for $|\arg \lambda| \leq \pi-\varphi$ belonging to the space $W_{p}^{m}\left(0, b ; E\left(A^{m}\right), E\right)$ has the form

$$
u(x)=\sum_{n=1}^{d} e^{x \omega_{n} A_{\lambda}} g_{n}+\sum_{n=d+1}^{m} e^{-(b-x) \omega_{n} A_{\lambda}} g_{n} .
$$

Let

$$
V_{n \lambda}(x)= \begin{cases}e^{x \omega_{n} A_{\lambda}} & \text { for } n=1,2, \ldots, d, \\ e^{-(b-x) \omega_{n} A_{\lambda}} & \text { for } n=d+1, d+2, \ldots, m .\end{cases}
$$

Now, taking into account the boundary conditions (2.29), we obtain algebraic linear equations with respect to $g_{1}, g_{2}, \ldots, g_{m}$, that is,

$$
\sum_{n=1}^{m} L_{k}\left(V_{\lambda n}\right) g_{n}=f_{n}, \quad n=1,2, \ldots, m
$$

where

$$
\begin{aligned}
& L_{k}\left(V_{\lambda n}\right)=\sum_{i=0}^{v_{k}}\left(-\omega_{n}\right)^{i}\left[\alpha_{k i}+\beta_{k i} e^{b \omega_{n} A_{\lambda}}+\sum_{j=1}^{N_{k}} \delta_{k j i} e^{x_{k j t} \omega_{n} A_{\lambda}}\right] A_{\lambda}^{i} \quad \text { for } n=1,2, \ldots, d, \\
& L_{k}\left(V_{\lambda n}\right)=\sum_{i=0}^{\nu_{k}} \omega_{n}^{i}\left[\alpha_{k i} e^{-b \omega_{n} A_{\lambda}}+\beta_{k}+\sum_{j=1}^{N_{k}} \delta_{k j i} e^{-\left(b-x_{k j t}\right) \omega_{n} A_{\lambda}}\right] A_{\lambda}^{i} \quad \text { for } n=d+1, \ldots, m .
\end{aligned}
$$

We obtain from the above equalities that the determinant of the system (3.8) is the determinant-operator

$$
D(\lambda, t)=\operatorname{det}\left[L_{k}\left(V_{\lambda n}\right)\right]=[\omega(t) I+B(\lambda, t)] A_{\lambda}^{\nu_{0}}
$$

where $\nu_{0}=\sum_{n=1}^{m} \nu_{n}$ and $B(\lambda, t)$ is an operator in $E$ in which all elements are bounded operators containing $A_{\lambda}^{-\gamma}$ for some $\gamma>0$ and bounded operators $V_{n \lambda}(0), V_{n \lambda}(b), V_{n \lambda}\left(x_{k j t}\right)$. Therefore, by virtue of the properties of positive operators and holomorphic semigroups (see [38, Section 1.13.1]) and in view of continuity of the functions $\alpha_{k i}, \beta_{k i}, \delta_{k j i}$ on $\bar{\Omega}$, it is easy to see that for $|\arg \lambda| \leq \pi-\varphi,|\lambda| \rightarrow \infty$ we have $\|B(\lambda, t)\|_{B\left(E^{2}\right)} \rightarrow 0$ uniformly with respect to $t \in \bar{\Omega}$. Therefore, by the condition $\omega(t) \neq 0$ for $|\arg \lambda| \leq \pi-\varphi, \lambda \rightarrow \infty$, the operator $Q(\lambda, t)[\omega(t) I+B(\lambda, t)]^{-1}$ is uniformly invertible in $E$ with respect to $\lambda$ and $t$, that is,

$$
\|Q(\lambda, t)\| \leq C
$$

Therefore, the operator $D(\lambda, t)$ is invertible in $E$, and the inverse operator $D^{-1}(\lambda, t)$ can be expressed in the form

$$
D^{-1}(\lambda, t)=A_{\lambda}^{-v_{0}} Q(\lambda, t)
$$


22 Maximal regular BVPs in Banach-valued weighted space

Thus, $D^{-1}(\lambda, t)$ is uniformly bounded with respect to the parameters $\lambda$ and $t$, that is,

$$
\left\|D^{-1}(\lambda, t)\right\| \leq C
$$

Consequently, system (3.8) has a unique solution for $|\arg \lambda| \leq \pi-\varphi$ and $|\lambda|$ sufficiently large, and the solution can be expressed in the form

$$
g_{n}=Q(\lambda, t) \sum_{i=1}^{m} C_{n i}(\lambda, t) A_{\lambda}^{-v_{n}} f_{i}, \quad n=1,2, \ldots, m,
$$

where $C_{n i}(\lambda, t)$ are operators in $E$ involving linear combinations of uniformly, with respect to $\lambda$ and $t$, bounded operators $A_{\lambda}^{-\gamma}, \gamma>0$ and $V_{n \lambda}(0), V_{n \lambda}(b), V_{n \lambda}\left(x_{k j t}\right)$. Substituting (3.14) into (3.6), we obtain a representation of the solution of the problem (3.1), (2.29) as

$$
u(x)=Q(\lambda, t)\left[\sum_{n=1}^{m} \sum_{i=1}^{m} C_{n i}(\lambda, t) V_{\lambda_{n}}(x) A_{\lambda}^{-v_{n}} f_{i}\right]
$$

Since $b=b(t), \alpha_{k i}=\alpha_{k i}(t), \delta_{k i j}=\delta_{k i j}(t)$ are continuous functions on $\bar{\Omega}$, by virtue of properties of holomorphic semigroups (see [38, Section 1.13.1]), in view of (3.13) and by virtue of uniform boundedness of the operators $C_{n i}$ and $Q(\lambda, t)$ with respect to $\lambda$ and $t$, for $|\arg \lambda| \leq \pi-\varphi, t \in \bar{\Omega}$, and for sufficiently large $|\lambda|$, we obtain

$$
\begin{aligned}
& \sum_{k, n=0}^{m}(1+|\lambda|)^{m-k-n}\left\|A^{k} u^{(n)}\right\|_{L_{p}(0, b ; E)} \\
& \quad \leq C\|Q(\lambda, t)\|\left\{\sum_{k, n=0}^{m}(1+|\lambda|)^{m-k-n} \sum_{j=1}^{m} \sum_{i=1}^{m}\left(\int_{0}^{b}\left\|A_{\lambda}^{k+n-v_{j}} V_{n \lambda}(x) f_{i}\right\|^{p} d x\right)^{1 / p}\right\} .
\end{aligned}
$$

Using the equality $A_{\lambda}^{k+n-v_{j}}=A_{\lambda}^{-(m-k-n)} A_{\lambda}^{m-v_{j}}$ and Theorem 2.6, we obtain from (3.16)

$$
\begin{aligned}
& \sum_{k, n=0}^{m}(1+|\lambda|)^{m-k-n}\left\|A^{k} u^{(n)}\right\|_{L_{p}(0, b ; E)} \\
& \quad \leq C \sum_{k, n=0}^{m}(1+|\lambda|)^{m-k-n}\left\|A_{\lambda}^{-(m-k-n)}\right\| \sum_{i=1}^{m}\left[\int_{0}^{b}\left\|A_{\lambda}^{m-\gamma_{i}} V_{n \lambda}(x) f_{i}\right\|^{p} d x\right]^{1 / p} \\
& \quad \leq C \sum_{i=1}^{m}\left[\left\|f_{i}\right\|_{E_{k}}+|\lambda|^{1-\theta_{i}}\left\|f_{i}\right\|_{E}\right]
\end{aligned}
$$

where the constant $C$ is independent of the parameters $\lambda$ and $t$. Therefore, we obtain the estimate (3.5).

\section{Nonhomogeneous equations}

Now, we consider nonlocal boundary value problems for nonhomogeneous equations of the form (2.28), (2.29), where $A_{\lambda}=A+\lambda, A$ is, generally speaking, an unbounded 
operator in $E$ and $b=b(t), \alpha_{k i}=\alpha_{k i}(t), \beta_{k i}=\beta_{k i}(t), \delta_{k i j}=\delta_{k i j}(t)$ are complex-valued functions on $\bar{\Omega}$. Let $E_{k}=\left(E\left(A^{m}\right), E\right)_{\theta_{k}, p}$, where $\theta_{k}=\left(\nu_{k}+1 / p\right) / m, k=1,2, \ldots, m$.

THeOREM 4.1. Let all conditions of Theorem 3.1 be satisfied and let $E$ be a Banach space satisfying a multiplier condition with respect to $p \in(1, \infty)$. Then, the operator $B_{0}: u \rightarrow B_{0} u=$ $\left\{L u, L_{1} u, L_{2} u, \ldots, L_{m} u\right\}$ for $|\arg \lambda| \leq \pi-\varphi$ and sufficiently large $|\lambda|$ is an isomorphism

$$
\text { from } W_{p}^{m}\left(0, b ; E\left(A^{m}\right), E\right) \quad \text { onto } \quad L_{p}(0, b ; E)+\sum_{k=1}^{m} E_{k} \text {. }
$$

Moreover, coercive uniformity with respect to $\lambda$ and $t$, that is,

$$
\begin{aligned}
& \sum_{k, n=0}^{m}(1+|\lambda|)^{m-k-n}\left\|A^{k} u^{(n)}\right\|_{L_{p}(0, b ; E)} \\
& \quad \leq C\left[\|f\|_{L_{p}(0, b ; E)}+\sum_{k=1}^{m}\left(\left\|f_{k}\right\|_{E_{k}}+|\lambda|^{1-\theta_{k}}\left\|f_{k}\right\|_{E}\right)\right]
\end{aligned}
$$

holds for the solution of (2.28), (2.29), where $k+n \leq m$.

Proof. By definition of the space $W_{p}^{m}\left(0, b ; E\left(A^{m}\right), E\right)$ and by virtue of the trace theorem in it (see [24] or [38, Section 1.8]), we obtain that the operator $u \rightarrow B_{0} u$ is bounded from $W_{p}^{m}\left(0, b ; E\left(A^{m}\right), E\right)$ onto $L_{p}(0, b ; E)+\sum_{k=1}^{m} E_{k}$. Then, by Banach's theorem it suffices to show that this operator is bijective. We have proved the uniqueness of the solution of the problem (2.28), (2.29) in Theorem 3.1. Therefore, we need only to prove that the problem (2.28), (2.29) for all $f \in L_{p}(0, b ; E)$ has a solution satisfying estimate (4.2). We define

$$
f_{t}(x)= \begin{cases}f(x) & \text { if } x \in[0, b(t)] \\ 0 & \text { if } x \notin[0, b(t)]\end{cases}
$$

We now show that the solution of the problem (2.28), (2.29) belonging to the space $W_{p}^{m}\left(0, b ; E\left(A^{m}\right), E\right)$ can be represented as a sum $u(x)=u_{1}(x)+u_{2}(x)$, where $u_{1}$ is the restriction on $[0, b]$ of the solution $\tilde{u}_{1}$ of the equation

$$
L u=f_{t}(x), \quad x \in \mathbb{R}=(-\infty, \infty),
$$

and $u_{2}$ is a solution of the problem

$$
L u=0, \quad L_{k} u=f_{k}-L_{k} u_{1}, \quad k=1,2, \ldots, m
$$

A solution of (4.4) is given by the formula

$$
\tilde{u}_{1}(x)=F^{-1} L^{-1}(\lambda, \xi) F f_{t}=\frac{1}{2 \pi} \int_{-\infty}^{\infty} e^{i \xi x} L^{-1}(\lambda, \xi)\left(F f_{t}\right)(\xi) d \xi
$$


24 Maximal regular BVPs in Banach-valued weighted space

where $F f_{t}$ is the Fourier transform of the function $f_{t}$ and $L(\lambda, \xi)$ is a characteristic operator pencil of (4.4), that is,

$$
L(\lambda, \xi)=\sum_{k=0}^{m} a_{k}(i \xi)^{k} A_{\lambda}^{m-k}
$$

It follows from the above expression that

$$
\begin{aligned}
& \sum_{k, n=0}^{m}(1+|\lambda|)^{m-k-n}\left\|A^{m-k} \tilde{u}_{1}^{(n)}\right\|_{L_{p}(R ; E)} \\
& \quad=\sum_{k, n=0}^{m}(1+|\lambda|)^{m-k-n}\left\|F^{-1}(i \xi)^{n} L^{-1}(\lambda, i \xi) A^{m-k} F f_{t}\right\|_{L_{p}(R ; E)},
\end{aligned}
$$

where $k+n \leq m$. We show that the operator-valued functions

$$
H(\lambda, \xi)=\lambda L^{-1}(\lambda, i \xi), \quad H_{n k}(\lambda, i \xi)=(1+|\lambda|)^{m-k-n}(\xi)^{n} A^{k} L^{-1}(\lambda, i \xi),
$$

$n, k=0,1, \ldots, m, n+k \leq m$, are Fourier multipliers in $L_{p}(R ; E)$, uniformly with respect to the parameter $\lambda$. Conditions (2) and (4) imply

$$
\lambda-i \omega_{k}^{-1} \xi \in S(\varphi), \quad L^{-1}(\lambda, i \xi)=\prod_{k=1}^{m}\left(i \xi-\omega_{k} A_{\lambda}\right)^{-1}
$$

Then by virtue of the resolvent properties of the positive operator $A$, we obtain

$$
|\lambda||| L^{-1}(\lambda, i \xi)|| \leq C
$$

and

$$
\begin{aligned}
\left\|(1+|\lambda|)^{m-n-k} \xi^{n} A^{k} L^{-1}(\lambda, i \xi)\right\| \\
\quad=\left\|(1+|\lambda|)^{m-n-k} \xi^{n} A^{k} \prod_{j=1}^{m}\left(i \xi-\omega_{j} A_{\lambda}\right)^{-1}\right\| \\
\quad \leq\left\|\prod_{j=1}^{n} \xi\left(i \xi-\omega_{j} A_{\lambda}\right)^{-1}\right\|\left\|\prod_{j=1}^{k} A\left(i \xi-\omega_{j} A_{\lambda}\right)^{-1}\right\|\|\|_{j=1}^{m-n-k}(1+|\lambda|)\left(i \xi-\omega_{j} A_{\lambda}\right)^{-1} \| \\
\quad \leq C
\end{aligned}
$$

for $n, k=0,1, \ldots, m, n+k \leq m$. Therefore, using (4.9), we obtain

$$
\|H(\lambda, \xi)\| \leq C, \quad\left\|H_{n k}(\lambda, \xi)\right\| \leq C .
$$


Since

$$
\begin{aligned}
\frac{d}{d \xi} H(\lambda, \xi)= & -L^{-1}(\lambda, i \xi) \frac{d}{d \xi} L(\lambda, i \xi) L^{-1}(\lambda, i \xi), \\
\frac{d}{d \xi} H_{n k}(\lambda, t, \xi)= & (1+|\lambda|)^{m-k-n} n \xi^{n-1} A^{k} L^{-1}(\lambda, i \xi) \\
& -\xi^{n} A^{k} L^{-1}(\lambda, i \xi) \frac{d}{d \xi} L(\lambda, t, i \xi) L^{-1}(\lambda, i \xi),
\end{aligned}
$$

and using (4.13) for all $\xi \in \mathbb{R} \backslash\{0\}$, we obtain

$$
\left\|\frac{d}{d \xi} H(\lambda, \xi)\right\| \leq C|\xi|^{-1}, \quad\left\|\frac{d}{d \xi} H_{n k}(\lambda, \xi)\right\| \leq|\xi|^{-1}
$$

for $n, k=0,1, \ldots, m, n+k \leq m$. It is easy to see that due to $R$-positivity of the operator $A$, the operator-valued functions $H(\lambda, \xi)$ and $H_{n k}(\lambda, \xi)$ are $R$-bounded with $R$-bound independent of $\lambda$. Moreover, it is easy to see from the inequalities (4.13) that the operatorvalued functions $\xi(d / d \xi) H(\lambda, \xi)$ and $\xi(d / d \xi) H_{n k}(\lambda, \xi)$ are $R$-bounded with $R$-bound independent of $\lambda$ and $t$ (or applying [39, Lemma 3.8] we can get this for UMD spaces). Then, in view of Definition 1.1 it follows from (4.9) and (4.15) that the functions $H(\lambda, \xi)$ and $H_{k}(\lambda, \xi)$ are Fourier multipliers in $L_{p, \gamma}(R ; E)$, uniformly with respect to the parameter $\lambda$. Then, by using (4.8), we get

$$
\sum_{k, n=0}^{m}(1+|\lambda|)^{m-k-n}\left\|\xi^{n} A^{m-k} F \tilde{u}_{1}\right\|_{p} \leq C\left\|\hat{f}_{t}\right\|_{p}, \quad k+n \leq m,
$$

uniformly with respect to $\lambda$ and $t$. Then, we have

$$
\tilde{u}_{1} \in W_{p}^{m}\left(R ; E\left(A^{m}\right), E\right)
$$

By virtue of (4.5) (or [38, Section 1.8]), we get that $u_{1}^{\left(\nu_{k}\right)}(\cdot) \in E_{k}, k=1,2, \ldots, m$. Hence, $L_{k} u_{1} \in E_{k}$. Thus, by Theorem 3.1 and due to $\theta_{k} \leq \eta_{k}$ for $k=1,2, \ldots, m$, the problem (4.5) has a unique solution $u_{2}(x)$ that belongs to the space $W_{p}^{m}\left(R ; E\left(A^{m}\right), E\right)$ for $|\arg \lambda| \leq \pi-\varphi$ and for sufficiently large $|\lambda|$. Moreover, for a solution of the problem (4.5), we have

$$
\begin{aligned}
& \sum_{k, n=0}^{m}(1+|\lambda|)^{m-k-n}\left\|A^{k} u_{2}^{(n)}\right\|_{p} \\
& \leq C \sum_{k=1}^{m}\left(\left\|f_{k}-L_{0 k} u_{1}\right\|_{E_{k}}+|\lambda|^{1-\theta_{k}}|| f_{k}-L_{0 k} u_{1} \|_{E}\right) \\
& \leq C \sum_{k=1}^{m}\left[\left\|f_{k}\right\|_{E_{k}}+|\lambda|^{1-\theta_{k}}|| f_{k}\left\|_{E}+|\lambda|^{1-\theta_{k}}|| L_{0, k} u_{1}\right\|_{E}\right. \\
& \left.\quad+\left\|u_{1}^{\left(\nu_{k}\right)}\right\|_{C\left([0, b], E_{k}\right)}+\left|\lambda^{1-\theta_{k}}\right|\|u\|_{C([0, b] ; E)}\right] .
\end{aligned}
$$


26 Maximal regular BVPs in Banach-valued weighted space

From (4.16) we obtain

$$
\sum_{k, n=0}^{m}(1+|\lambda|)^{m-k-n}\left\|A^{k} u_{1}^{(n)}\right\|_{p} \leq C\|f\|_{p}
$$

Therefore, by virtue of [24] (or [38, Section 1.8]) and the estimate (4.19), we obtain

$$
\left\|u_{1}^{\left(\nu_{k}\right)}(\cdot)\right\|_{E_{k}} \leq C\left\|u_{1}\right\|_{W_{p}^{m}\left(0, b ; E\left(A^{m}\right), E\right)} \leq C\|f\|_{p}
$$

By Theorem 2.7 for $\mu \in \mathbb{C}, u \in W_{p}^{m}(0, b ; E)$, we get

$$
|\mu|^{2-v_{k}}\left\|u^{\left(v_{k}\right)}(\cdot)\right\| \leq C\left[|\mu|^{1 / p}\|u\|_{W_{p}^{m}(0, b ; E)}+|\mu|^{2+1 / p}\|u\|_{p}\right] .
$$

Dividing by $|\mu|^{1 / p}$ and substituting $\lambda=\mu^{2}$ for $\lambda \in \mathbb{C}$ and $u \in W_{p}^{m}(0, b ; E)$, from $(4.21)$ we get

$$
|\lambda|^{1-\theta_{k}}\left\|u^{\left(\nu_{k}\right)}(\cdot)\right\| \leq C\left[\|u\|_{W_{p}^{m}(0, b ; E)}+|\lambda|\|u\|_{p}\right]
$$

From (4.19), (4.20), and (4.22), we obtain

$$
|\lambda|^{1-\theta_{k}}\left\|u_{1}^{\left(\nu_{k}\right)}(\cdot)\right\| \leq C\left[\left\|u_{1}\right\|_{W_{p}^{m}(0, b ; E)}+|\lambda|\left\|u_{1}\right\|_{p}\right] \leq C\|f\|_{p}
$$

uniformly with respect to the parameters $t$ and $\lambda$. Similarly, we get for $k=1,2, \ldots, m$

$$
|\lambda|^{1-\theta_{k}}\left\|u_{1}^{\left(\nu_{k}\right)}\left(x_{k j i}\right)\right\| \leq C\left[\left\|u_{1}\right\|_{W_{p}^{m}\left(0, b ; E\left(A^{m}\right), E\right)}+|\lambda|\left\|u_{1}\right\|_{p}\right] \leq C\|f\|_{p}
$$

Hence, from the estimates (4.18), (4.20) and (4.23), (4.24) for $|\arg \lambda| \leq \pi-\varphi,|\lambda| \rightarrow \infty$ and $t \in \bar{\Omega}$, we obtain

$$
\sum_{k, n=0}^{m}(1+|\lambda|)^{m-k-n}\left\|A^{k} u_{2}^{(n)}\right\|_{p} \leq C\left[\|f\|_{p}+\sum_{k=1}^{m}\left(\left\|f_{k}\right\|_{E_{k}}+|\lambda|^{1-\theta_{k}}\left\|f_{k}\right\|_{E}\right)\right] .
$$

Then, the estimates (4.19) and (4.25) imply (4.2).

Remark 4.2. Let the boundary conditions (2.29) be homogeneous, that is, $f_{k}=0$. We consider a differential operator $Q_{\lambda}$ acting in $L_{p}(0, b ; E)$ and generated by the problem (2.28), (2.29), that is,

$$
\begin{gathered}
D\left(Q_{\lambda}\right)=W_{p}^{m}\left(0, b ; E(A), E, L_{k}\right), \\
Q_{\lambda} u=\sum_{k=0}^{m} a_{k} A_{\lambda}^{m-k} u^{(k)}(x), \quad x \in(0, b(t)), t \in \Omega .
\end{gathered}
$$

Then, by Theorem 4.1, for $|\arg \lambda| \leq \pi-\varphi$ and sufficiently large $|\lambda|$, the operator $Q_{\lambda}$ has a bounded inverse operator from the space $L_{p}(0, b ; E)$ to the space $W_{p}^{m}\left(0, b ; E\left(A^{m}\right), E\right)$, and 
for all solution of this problem we have

$$
\begin{gathered}
\left\|Q_{\lambda_{0}} u\right\|_{L_{p}(0, b ; E)}=\|u\|_{W_{p}^{m}\left(0, b ; E\left(A^{m}\right), E\right)}, \\
\sum_{k, n=0}^{m}(1+|\lambda|)^{m-k-n}\left\|A^{k} u^{(n)}\right\|_{L_{p}(0, b ; E)} \leq C\|f\|_{L_{p}(0, b ; E)}, \quad k+n \leq m .
\end{gathered}
$$

We now consider a boundary value problem (2.28), (2.29) with $a_{k}=0$ for $k=1,2, \ldots$, $m-1, a_{m}=1$, and $f_{k}=0$ for $k=1,2, \ldots, m$, that is,

$$
L u=a_{0} u^{(m)}(x)+A_{\lambda} u(x)=f(x), \quad x \in(0, b), \quad L_{k} u=0, \quad k=1,2, \ldots, m,
$$

where $L_{k}$ are defined in (2.29) and where $A_{\lambda}=A+\lambda, A$ is, generally speaking, an unbounded operator in $E$ and $b=b(t), \alpha_{k i}=\alpha_{k i}(t), \beta_{k i}=\beta_{k i}(t), \delta_{k i j}=\delta_{k i j}(t)$ are complexvalued functions on $\bar{\Omega}$. By $B$ we will denote a differential operator acting in $F=L_{p}(0, b ; E)$ and generated by the problem (4.28), that is, it is defined by

$$
\begin{gathered}
D(B)=W_{p}^{m}\left(0, b ; E(A), E, L_{k}\right), \\
B u=a_{0} u^{(m)}(x)+A u(x), \quad x \in(0, b(t)), t \in \Omega .
\end{gathered}
$$

Let $\omega_{j}$ be the roots of the equation

$$
a_{0} \omega^{m}+1=0
$$

Theorem 4.1 implies the following result.

Corollary 4.3. Let the following conditions be satisfied:

(1) $E$ is a Banach space satisfying a multiplier condition with respect to $p \in(1, \infty)$;

(2) $A$ is a $\varphi$-positive operator in $E$ and $0<\varphi \leq \pi$;

(3) $\omega(t) \neq 0$ for all $t \in \Omega$ and $\theta_{k}=\nu_{k} / m+1 / m p, p \in(1, \infty)$;

(4) $a_{0} \neq 0$ and $\left|\arg \omega_{j}-\pi\right| \leq \pi / 2-\varphi, j=1,2, \ldots, d,\left|\arg \omega_{j}\right| \leq \pi / 2-\varphi, j=d+1, \ldots$, $m, 0<d<m$;

(5) $\alpha_{k i}, \beta_{k i}, \delta_{k j i}$ are continuous functions on $\bar{\Omega}$.

Then, for $|\arg \lambda| \leq \pi-\varphi$, there exists a resolvent $(B-\lambda I)^{-1}$ of the operator $B$, and coercive uniformity with respect to $\lambda$ and $t$, that is,

$$
\sum_{k, n=0}^{m}(1+|\lambda|)^{1-(k+n) / m}\left\|A^{k / m} \frac{d^{n}}{d x^{n}}(B+\lambda)^{-1}\right\|_{L(F)} \leq C, \quad k+n \leq m,
$$

holds. Moreover,

$$
\left\|B_{\lambda_{0}} u\right\|_{L_{p}(0, b ; E)}=\|u\|_{W_{p}^{m}(0, b ; E(A), E)} .
$$

Proof. By [22, Theorems 10.6 and 10.3], $A=\left(A^{1 / m}\right)^{m}$, and the operator $A^{1 / m}, m \geq 2$, is $\varphi$ positive in $E$ for $0<\varphi \leq \pi$. Then, in view of Theorem 4.1, the problem (4.28) is coercive in $L_{p}(0, b ; E)$, uniformly with respect to $t \in \bar{\Omega}$, which in turn implies that the operator $B_{\lambda}$ 
28 Maximal regular BVPs in Banach-valued weighted space

for $|\arg \lambda| \leq \pi-\varphi$ and for sufficiently large $|\lambda|$ has a bounded inverse operator $(B+\lambda)^{-1}$ from $L_{p}(0, b ; E)$ to $W_{p}^{m}(0, b ; E(A), E)$, and the relations (4.31) and (4.32) hold.

\section{BVPs for anisotropic partial DOEs}

We consider a principal part of the problem (2.31), (2.32), that is, the boundary value problem

$$
\begin{aligned}
L_{0} u & :=a_{1} D_{x}^{l_{1}} u(x, y)+a_{2} D_{y}^{l_{2}} u(x, y)+A_{\lambda} u(x, y)=f(x, y), \\
L_{01 j} u & =0, \quad j=1,2, \ldots, l_{1}, \quad L_{02 j} u=0, \quad j=1,2, \ldots, l_{2},
\end{aligned}
$$

where

$$
\begin{aligned}
L_{01 j} u & :=\sum_{i=0}^{m_{1 j}}\left[\alpha_{1 j i} u^{(i)}(0, y)+\beta_{1 j i} u^{(i)}(1, y)+\sum_{\nu=1}^{N_{1 j}} \delta_{1 j i v} u^{(i)}\left(x_{j v}, y\right)\right]=0, \quad j=1,2, \ldots, l_{1}, \\
L_{02 j} u & :=\sum_{i=0}^{m_{2 j}}\left[\alpha_{2 j i} u^{(i)}(x, 0)+\beta_{2 j i} u^{(i)}(x, h)+\sum_{\nu=1}^{N_{2 j}} \delta_{2 j i v} u^{(i)}\left(x, \Gamma_{j v}\right)\right]=0, \quad j=1,2, \ldots, l_{2},
\end{aligned}
$$

in $E$. By $\omega_{k j}, j=1,2, \ldots, l_{k}, k=1,2$, we denote the roots of the equations

$$
a_{k} \omega^{l_{k}}+1=0, \quad k=1,2
$$

Let $\left[v_{k j i}\right]_{i, j=1,2, \ldots, l_{k}}, k=1,2$, be $l_{k}$-dimensional matrices with determinant $\eta_{k}=\operatorname{det}\left[v_{k j i}\right]$, where

$$
v_{k j i}= \begin{cases}\alpha_{k j}\left(-\omega_{i}\right)^{m_{k j}} & \text { if } i=1,2, \ldots, d_{k} \\ \beta_{k j} \omega_{i}^{m_{k j}} & \text { if } i=d_{k}+1, d_{k}+2, \ldots, l_{k}\end{cases}
$$

$0<d_{k}<l_{k}, j=1,2, \ldots, l_{k}, k=1,2$. Let

$$
\gamma_{1}(x)=x^{\gamma_{1}}, \quad \gamma_{1}(y)=y^{\gamma_{2}}
$$

THEOREm 5.1. Assume that the following conditions are satisfied:

(1) $E$ is a Banach space satisfying a multiplier condition with respect to $p \in(1, \infty)$ and with respect to the weight function $\gamma(x, y)=x^{\gamma_{1}} y^{\gamma_{2}}, 0 \leq \gamma_{1}, \gamma_{2}<1-1 / p$;

(2) $A$ is an R-positive operator in $E$ for $0<\varphi \leq \pi$;

(3) $\eta_{k}=\operatorname{det}\left[v_{k j i}\right] \neq 0$ for $k=1,2$;

(4) $a_{k} \neq 0$ and $\left|\arg \omega_{k j}-\pi\right| \leq \pi / 2-\varphi, j=1,2, \ldots, d_{k},\left|\arg \omega_{k j}\right| \leq \pi / 2-\varphi, j=d_{k}+$ $1, \ldots, l_{k}, 0<d_{k}<l_{k}, k=1,2$. 
Then, the problem (5.1), (5.2) for $f \in L_{p, \gamma}(G ; E), p \in(1, \infty),|\arg \lambda| \leq \pi-\varphi$, and sufficiently large $|\lambda|$, has a unique solution that belongs to the space $W_{p, \gamma}^{l}(G ; E(A), E)$, and coercive uniformity with respect to $t$ and $\lambda$, that is,

$$
\begin{aligned}
\sum_{j=0}^{l_{1}}(1+ & |\lambda|)^{1-j / l_{1}}\left\|D_{x}^{j} u\right\|_{L_{p, \gamma}(G ; E)} \\
& +\sum_{j=0}^{l_{2}}(1+|\lambda|)^{1-j / l_{2}}\left\|D_{y}^{j} u\right\|_{L_{p, \gamma}(G ; E)}+\|A u\|_{L_{p, \gamma}(G ; E)} \\
\leq & M\|f\|_{L_{p, \gamma}(G ; E)}
\end{aligned}
$$

holds for the solution of the problem (5.1), (5.2).

Proof. We first consider a nonlocal boundary problem

$$
\begin{gathered}
L u=a_{1} u^{\left(l_{1}\right)}(x)+A_{\lambda} u(x)=f(x), \quad x \in(0,1), \\
L_{01 j} u=\sum_{i=0}^{m_{1 j}}\left[\alpha_{1 j i} u^{(i)}(0)+\beta_{1 j i} u^{(i)}(1)+\sum_{k=1}^{N_{1 j}} \delta_{1 j i k} u^{(i)}\left(x_{k j}\right)\right]=0
\end{gathered}
$$

in $L_{p, \gamma_{1}}(0,1 ; E)$, where $A$ is a positive operator in $E$ and $x_{k j} \in(0,1), \alpha_{1 j i}, \beta_{1 j i}, \delta_{1 j i}$ are complex numbers. By [18, Theorem 10.6], we have $A_{\lambda}=\left(A_{\lambda}^{1 / l_{1}}\right)^{l_{1}}$. Then, by using a similar technique to that in Theorem 4.1, we obtain that for all $f \in L_{p, \gamma_{1}}(0,1 ; E),|\arg \lambda| \leq \pi-\varphi$ and sufficiently large $\lambda$, the problem (5.8) has a unique solution that belongs to the space $W_{p, \gamma_{1}}^{l_{1}}(0,1 ; E(A), E)$, and coercive uniformity with respect to $\lambda$, that is,

$$
\sum_{i, j=0}^{l_{1}}(1+|\lambda|)^{1-(i+j) / l_{1}}\left\|A^{i / l_{1}} u^{(j)}\right\|_{p, \gamma_{1}} \leq M\|f\|_{p, \gamma_{1}}, \quad i+j \leq l_{1}
$$

holds for the solution of the problem (5.8). We now consider in $L_{p, \gamma}(G ; E)$ the boundary value problem (5.1), (5.2). This problem can be expressed as

$$
\begin{gathered}
D_{y}^{l_{2}} u(y)+B u(y)+\lambda u(y)=f(y), \\
L_{02 j} u=\sum_{i=0}^{m_{2 j}}\left[\alpha_{2 j i} u^{(i)}(0)+\beta_{2 j i} u^{(i)}(h)+\sum_{\nu=1}^{N_{2 j}} \delta_{2 j i v} u^{(i)}\left(\Gamma_{j v}\right)\right]=0, \quad j=1,2, \ldots, l_{2},
\end{gathered}
$$

where $B$ is a differential operator acting in $L_{p, \gamma_{1}}(0,1 ; E)$ and generated by the problem (5.8), that is,

$$
\begin{gathered}
D(B)=W_{p}^{l_{1}}\left(0,1 ; E(A), E, L_{01 j}\right), \\
B u=a_{1} u^{\left(l_{1}\right)}(x)+A u(x), \quad x \in(0,1) .
\end{gathered}
$$


Then, by virtue of Corollary 4.3 and in view of (5.9), we obtain that the operator $B$ is positive in $F=L_{p, \gamma_{1}}(0,1 ; E)$ and

$$
\begin{gathered}
\sum_{k, n=0}^{l_{1}}(1+|\lambda|)^{1-(k+n) / l_{1}}\left\|A^{k / l_{1}} \frac{d^{n}}{d x^{n}}(B+\lambda)^{-1}\right\|_{L(F)} \leq C, \quad k+n \leq l_{1}, \\
\left\|B_{\lambda_{0}} u\right\|_{F}=\|u\|_{W_{P, \gamma_{1}}^{l_{1}}(0,1 ; E(A), E)}
\end{gathered}
$$

Using Theorem 4.1 and Remark 4.2, the problem (5.10) for

$$
f \in L_{p, \gamma_{2}}\left(0, h ; L_{p, \gamma_{1}}(0,1 ; E)\right)=L_{p, \gamma}(G ; E), \quad|\arg \lambda| \leq \pi-\varphi
$$

and sufficiently large $|\lambda|$, has a unique solution $u \in W_{p, \gamma_{2}}^{l_{2}}\left(0, h ; E_{1}(B), E_{1}\right)$, and coercive uniformity with respect to $t$ and $\lambda$, that is,

$$
\sum_{i, j=0}^{l_{2}}(1+|\lambda|)^{1-(i+j) / l_{2}}\left\|B^{i / l_{2}} u^{(j)}\right\|_{L_{p, \gamma_{2}}\left(0, h ; E_{1}\right)} \leq M\|f\|_{L_{p, \gamma_{2}}\left(0, h ; E_{1}\right)}, \quad i+j \leq l_{2}
$$

holds for the solution of the problem (5.9). From (5.15), we obtain

$$
\|B u\|_{L_{p, \gamma}(G ; E)}+\sum_{j=0}^{l_{2}}(1+|\lambda|)^{1-j / l_{2}}\left\|D_{y}^{j} u\right\|_{L_{p, \gamma}(G ; E)} \leq M\|f\|_{L_{p, \gamma}(G ; E)} .
$$

Moreover, by Theorem 2.1, we have

$$
\|u\|_{W_{p, \gamma_{1}}^{l_{1}}(0,1 ; E(A), E)}=\|A u\|_{L_{p, \gamma_{1}}(0,1 ; E)}+\sum_{j=0}^{l_{1}}\left\|u^{(j)}\right\|_{L_{p, \gamma_{1}}(0,1 ; E)} \cdot
$$

Therefore, by virtue of (5.12) and (5.16), we have (5.7).

Let $F=L_{p, \gamma_{1}}(0,1 ; E)$ and $F_{0}=W_{p, \gamma_{1}}^{l_{1}}\left(0,1 ; E(A), E, L_{01 j}\right)$.

THEOREM 5.2. In addition to the conditions of Theorem 5.1 assume the following:

(1) $A_{\alpha}(x) A^{-(1-|\alpha: l|-\mu)} \in L_{\infty}(G ; L(E))$ for some $0<\mu \leq 1-|\alpha: l|$;

(2) if $m_{k j}=0$, then $T_{k j v}=0$, and if $m_{k j} \neq 0$, then for $\varepsilon>0$,

$$
\left\|T_{k j v} u\right\|_{\left(F_{0}, F\right)_{\left(1+p+\gamma_{2}\right) / p l_{2}, p}} \leq \varepsilon\|u\|_{\left(F_{0}, F\right)_{\left(1+\gamma_{2}\right) / p l_{2}, p}}+C(\varepsilon)\|u\|_{F}
$$

Then, problem (2.31), (2.32) for $f \in L_{p, \gamma}(G ; E), p \in(1, \infty),|\arg \lambda| \leq \pi-\varphi$ and for sufficiently large $|\lambda|$, has a unique solution that belongs to the space $W_{p, \gamma}^{l}(G ; E(A), E)$, and coercive uniformity with respect to $t$ and $\lambda$, that is (5.7) holds for the solution of the problem (2.31), (2.32). 
Proof. Let $u \in W_{p, \gamma}^{l}(G ; E(A), E)$ be a solution of the problem (2.31), (2.32). Then, $u=$ $u(x, y)$ is the solution of the problem

$$
\begin{gathered}
a_{1} D_{x}^{l_{1}} u(x, y)+a_{1} D_{y}^{l_{2}} u(x, y)+A_{\lambda} u(x, y)=f(x, y)-\sum_{|\alpha: l|<1} A_{\alpha}(x, y) D^{\alpha} u(x, y), \\
L_{01 j} u=L_{1 j} u-\sum_{\nu=1}^{M_{1 j}} T_{1 j \nu} u\left(x_{\nu 0}, y\right), \\
L_{02 j} u=L_{2 j} u-\sum_{\nu=1}^{M_{2 j}} T_{2 j \nu} u\left(x, \Gamma_{\nu 0}\right), \quad j=1, \ldots, l_{k}, k=1,2,
\end{gathered}
$$

where $L_{0 k j}$ are defined by (5.2). Let $Q_{0}$ and $Q$ be differential operators acting in $L_{p, \gamma}(G ; E)$ and generated by the boundary value problems (5.1), (5.2) and (2.31), (2.32), respectively. It is easy to see that

$$
W_{p, \gamma}^{l}(G ; E(A), E)=W_{p, \gamma_{2}}^{l_{2}}\left(0, h ; W_{p, \gamma_{1}}^{l_{1}}(0,1 ; E(A), E), L_{p, \gamma_{1}}(0,1 ; E)\right) .
$$

By virtue of [24] (or [38, Section 1.8]) and by Theorem 2.4, the operator $u \rightarrow u\left(x_{0}\right)$ is bounded from $W_{p, \gamma}^{l}(G ; E(A), E)$ into $\left(F_{0}, F\right)_{\left(1+\gamma_{2}\right) / p l_{2}, p}$ and

$$
\left\|u\left(x_{0 k}\right)\right\|_{\left(F_{0}, F\right)_{\left(1+\gamma_{2}\right) / p l_{2}, p}} \leq \varepsilon\|u\|_{W_{p, \gamma}^{l}(G ; E(A), E)}+C(\varepsilon)\|u\|_{L_{p, \gamma}(G ; E)} .
$$

Consequently, from condition (1) and by estimate (5.21), it follows for all $\varepsilon>0$ and $u \in$ $W_{p, \gamma}^{l}(G ; E(A), E)$ that

$$
\left\|T_{k j} u\right\|_{\left(F_{0}, F\right)_{\left(1+p+\gamma_{2}\right) / p l_{2}, p}} \leq \varepsilon\|u\|_{W_{p, \gamma}^{l}(G ; E(A), E)}+C(\varepsilon)\|u\|_{L_{p, \gamma}(G ; E)} .
$$

Then, by Theorem 5.1 and by the estimate (5.22) for all $u \in W_{p, \gamma}^{l}(G ; E(A), E),|\arg \lambda| \leq$ $\pi-\varphi$, and sufficiently large $|\lambda|$, we obtain

$$
\left\|T_{k j} u\right\|_{\left(F_{0}, F\right)_{\left(1+p+\gamma_{2}\right) / p l_{2}, p}} \leq \varepsilon\left\|\left(Q_{0}+\lambda\right) u\right\|_{p, \gamma}+C(\varepsilon)\|u\|_{p, \gamma} .
$$

It is clear that

$$
\|u\|_{p, \gamma}=\frac{1}{\lambda}\left\|\left(Q_{0}+\lambda\right) u-Q_{0} u\right\|_{p, \gamma}
$$

Moreover, for all $u \in W_{p, \gamma}^{l}(G ; E(A), E)$, we have

$$
\left\|Q_{0} u\right\|_{L_{p, \gamma}(G ; E)} \leq C\|u\|_{W_{p, \gamma}^{l}(G ; E(A), E)} .
$$


32 Maximal regular BVPs in Banach-valued weighted space

From (5.23), (5.24), (5.25) for $|\arg \lambda| \leq \pi-\varphi$ and sufficiently large $|\lambda|$, we obtain

$$
\left\|T_{k j} u\right\|_{\left(F_{0}, F\right)_{\left(1+p+\gamma_{2}\right) / p l_{2}, p}} \leq \varepsilon\left\|\left(Q_{0}+\lambda\right) u\right\|_{L_{p, \gamma}(G ; E)} .
$$

By Theorem 2.1 and by condition (1), for all $W_{p}^{l}(G ; E(A), E)$, we have

$$
\begin{aligned}
\sum_{|\alpha: l|<1}\left\|A_{\alpha}(x) D^{\alpha} u\right\|_{p, \gamma} & \leq C \sum_{|\alpha: l|<1}\left\|A^{1-|\alpha: l|-\mu} D^{\alpha} u\right\|_{p, \gamma} \\
& \leq \varepsilon\|u\|_{W_{p, \gamma}^{l}(G ; E(A), E)}+C(\varepsilon)\|u\|_{p, \gamma}
\end{aligned}
$$

where $\varepsilon$ is sufficiently small and $C(\varepsilon)$ is a continuous function. Using (5.24), (5.25), (5.27) for $|\arg \lambda| \leq \pi-\varphi$ and sufficiently large $|\lambda|$, we obtain

$$
\sum_{|\alpha: l|<1}\left\|A_{\alpha}(x) D^{\alpha} u\right\|_{p, \gamma} \leq \varepsilon\left\|\left(Q_{0}+\lambda\right) u\right\|_{p, \gamma}
$$

Then, by Theorem 5.1, by virtue of equality (2.31), and the estimates (5.26), (5.28), and using the perturbation theory of linear operators [19], we obtain the assertion.

THeOREM 5.3. Let all conditions of Theorem 5.2 be satisfied and let $A^{-1}$ be a compact operator in E. Then, the problem (2.31), (2.32) is Fredholm in $L_{p, \gamma}(G ; E)$.

Proof. By Theorem 5.2, the operator $Q+\lambda$ for $|\arg \lambda| \leq \pi-\varphi$ and sufficiently large $|\lambda|$ is Fredholm in $L_{p, \gamma}(G ; E)$. Moreover, by Theorem 2.2, the embedding

$$
W_{p, \gamma}^{l}(G ; E(A), E) \subset L_{p, \gamma}(G ; E)
$$

is compact. Since $Q u=(Q+\lambda) u-\lambda u$, by Theorem 5.2 and by the perturbation theory of linear operators [19], we obtain that the operator $Q$ is Fredholm in $L_{p, \gamma}(G ; E)$.

Let $Q$ be a differential operator generated by problem (2.31), (2.32). From Theorem 5.2, we obtain the following result.

Corollary 5.4. Let all conditions of Theorem 5.2 be satisfied. Then, for $|\arg \lambda| \leq \pi-\varphi$ and for sufficiently large $|\lambda|$ there exists the resolvent $(Q+\lambda)^{-1}$ in $E_{1}=L_{p, \gamma}(G ; E)$, and

$$
\begin{aligned}
\sum_{j=0}^{l_{1}}(1+ & |\lambda|)^{1-j / l_{1}}\left\|D_{x}^{j}(Q+\lambda)^{-1}\right\|_{B\left(E_{1}\right)} \\
& +\sum_{j=0}^{l_{2}}(1+|\lambda|)^{1-j / l_{2}}\left\|D_{y}^{j}(Q+\lambda)^{-1}\right\|_{B\left(E_{1}\right)}+\left\|A(Q+\lambda)^{-1}\right\|_{B\left(E_{1}\right)} \leq M .
\end{aligned}
$$


Remark 5.5. Theorems 5.1, 5.2, and 5.3 may be proved in a similar manner for the same nonlocal boundary value problem for differential-operator equations (1.2) on the same region $G \subset \mathbb{R}^{n}$, where $x=\left(x_{1}, x_{2}, \ldots, x_{n}\right), D_{k}^{i}=\partial^{i} / \partial x_{k}^{i}$.

\section{Spectral properties of anisotropic differential operators}

Consider an $n$-dimensional variant of the nonlocal boundary value problem (2.31), (2.32) on $G \subset \mathbb{R}^{n}$, that is,

$$
\begin{aligned}
L u= & \sum_{k=1}^{n} a_{k} D_{k}^{l_{k}} u(x)+\sum_{|\alpha: l|<1} A_{\alpha}(x) D^{\alpha} u(x)=f(x), \\
L_{k v_{k}} u= & \sum_{i=0}^{q_{k v_{k}}}\left[\alpha_{k v_{k} i} u^{(i)}\left(\Gamma_{k 0}\right)+\beta_{k v_{k} i} u^{(i)}\left(\Gamma_{k T}\right)+\sum_{j=1}^{N_{k v_{k}}} \delta_{k v_{k} i j} u^{(i)}\left(\Gamma_{k j d}\right)\right] \\
& +\sum_{j=1}^{M_{k v_{k}}} T_{k v_{k} j} u\left(\Gamma_{k j d 0}\right)=0,
\end{aligned}
$$

where

$$
\begin{aligned}
& G=\left\{x=\left(x_{1}, x_{2}, \ldots, x_{n}\right): 0<x_{k}<T_{k}\right\}, \quad 0 \leq q_{k v_{k}} \leq l_{k}-1, \\
& \Gamma_{k 0}=\left(x_{1}, \ldots, x_{k-1}, 0, \ldots, x_{n}\right), \quad \Gamma_{k T}=\left(x_{1}, \ldots, x_{k-1}, T_{k}, \ldots, x_{n}\right), \\
& \Gamma_{k j d}=\left(x_{1}, \ldots, x_{k-1}, d_{k j}, \ldots, x_{n}\right), \quad \Gamma_{k j d 0}=\left(x_{1}, \ldots, x_{k-1}, d_{k j 0}, \ldots, x_{n}\right) \text {, } \\
& d_{k j} \in\left(0, T_{k}\right), \quad d_{k j 0} \in\left[0, T_{k}\right] .
\end{aligned}
$$

Moreover, $A, A_{\alpha}$, and $T_{k v_{k} i j}$ are, generally speaking, unbounded operators in $E$. Let $Q$ denote a differential operator in $L_{p, \gamma}(G ; E)$ that is generated by the boundary value problem (6.1) and let $B_{p}=B\left(L_{p, \gamma}(G ; E)\right)$.

THeOREM 6.1. Suppose all conditions of Theorem 5.3 for $k=1,2, \ldots, n$ are satisfied and $E$ is a UMD space with base. Let

$$
\varkappa_{0}=\sum_{k=1}^{n} \frac{1}{l_{k}}<1, \quad s_{j}(I(E(A), E)) \sim j^{-1 / k_{0}}, \quad j \in \mathbb{N}, k_{0}>0 .
$$

Then, for $|\arg \lambda| \leq \pi-\varphi$ and sufficiently large $|\lambda|$ there exists a resolvent $(Q+\lambda)^{-1}$ of the operator $B$, and

$$
\begin{gathered}
\sum_{k=1}^{n} \sum_{j=0}^{l_{k}}(1+|\lambda|)^{1-j / l_{k}}\left\|D_{k}^{j}(Q+\lambda)^{-1}\right\|_{B_{p}}+\left\|A(Q+\lambda)^{-1}\right\|_{B_{p}} \leq M, \\
s_{j}\left((Q+\lambda)^{-1}\left(L_{p, \gamma}(G ; E)\right)\right) \sim j^{-1 /\left(k_{0}+\varkappa_{0}\right)} .
\end{gathered}
$$


34 Maximal regular BVPs in Banach-valued weighted space

Proof. By Theorem 5.2, the resolvent operator $(Q+\lambda)^{-1}$ exists and is bounded from $L_{p, \gamma}(G ; E)$ to $W_{p, \gamma}^{l}(G ; E(A), E)$. The first estimate in $(6.4)$ is obtained as in Corollary 5.4. Moreover, by Theorem 2.7, the embedding operator $I\left(W_{p, \gamma}^{l}(G ; E(A), E), L_{p, \gamma}(G ; E)\right)$ is compact and

$$
s_{j}\left(I\left(W_{p, \gamma}^{l}(G ; E(A), E), L_{p, \gamma}(G ; E)\right)\right) \sim j^{-1 /\left(k_{0}+\varkappa_{0}\right)} .
$$

Since

$$
\begin{aligned}
& (Q+\lambda)^{-1}\left(L_{p, \gamma}(G ; E)\right) \\
& \quad=(Q+\lambda)^{-1}\left(L_{p, \gamma}(G ; E), W_{p, \gamma}^{l}(G ; E(A), E)\right) I\left(W_{p, \gamma}^{l}(G ; E(A), E), L_{p, \gamma}(G ; E)\right),
\end{aligned}
$$

from the relations (6.5) and (6.6) we obtain the relation (6.4).

Theorem 6.2. Suppose all conditions of Theorem 6.1 are satisfied and let $\varphi \in(0, \pi / 2)$. Then, the root elements of the differential operator $Q$ are complete in $L_{p, \gamma}(G ; E)$.

Proof. The estimate (6.4) implies that the operator $Q+\lambda_{0}$ for sufficiently large $\lambda_{0}$ is $\varphi$ positive in $L_{p, \gamma}(G ; E)$. Moreover, from Theorem 2.7 we obtain that

$$
\left(Q+\lambda_{0}\right)^{-1} \in \sigma_{q}\left(L_{p, \gamma}(G ; E)\right), \quad q>\frac{1}{k_{0}+\varkappa_{0}} .
$$

Then, by virtue of [13, page 277] we obtain the assertion.

Remark 6.3. We consider in $L_{p}(0, b ; E)$ a degenerate boundary value problem

$$
\begin{aligned}
L u= & \sum_{k=0}^{m} a_{k} A_{\lambda}^{m-k} u^{[k]}(x)=f(x), \quad x \in(0, b), a_{m} \neq 0, \\
L_{k} u= & \sum_{i=0}^{v_{k}}\left[\alpha_{k i} u^{[i]}(0)+\beta_{k i} u^{(i)}(b(t))+\sum_{j=1}^{N_{k}} \delta_{k j i} u^{[i]}\left(x_{k j t}\right)\right] \\
& +\sum_{j=1}^{M_{k}} T_{k j} u\left(x_{k j t 0}\right)=f_{k}, \quad k=1,2, \ldots, m,
\end{aligned}
$$

in a Banach space $E$, on the varying region $0 \leq x \leq b(t)$, where $u^{[k]}(x)=\left(x^{\gamma}(d / d x)\right)^{k} u(x)$, $0 \leq \nu_{k} \leq m-1 ; \alpha_{k i}, \beta_{k i}, \delta_{k j i}$ are complex-valued functions depending on the domain parameters $t$ and $x_{k j t} \in(0, b(t)), x_{k j t 0} \in[0, b(t)]$ for $t \in \Omega$. Moreover, $A_{\lambda}=A+\lambda, A$ and $T_{k j}$ are, generally speaking, unbounded operators in $E$. 
Under the substitution

$$
y=(1-\gamma)^{-1} x^{1-\gamma},
$$

the spaces $L_{p}(0, b ; E)$ and $W_{p, \gamma}^{[2]}(0, b ; E(A), E)$ are mapped isomorphically onto the weighted spaces $L_{p, \tilde{\gamma}}(0, \tilde{b} ; E)$ and $W_{p, \tilde{\gamma}}^{2}(0, \tilde{b} ; E(A), E)$, respectively, where

$$
\tilde{b}=\frac{1}{1-\gamma}, \quad \tilde{\gamma}=(1-\gamma)^{\gamma /(1-\gamma)} y^{\gamma /(1-\gamma)}
$$

Moreover, under the substitution (6.10) the problem (1.1) reduces to the nondegenerate boundary value problem (2.28), (2.29). Consider in $L_{p}(G ; E)$ a degenerate boundary value problem of type (6.1) for the differential-operator equation

$$
\sum_{k=1}^{n} a_{k} D_{k}^{\left[l_{k}\right]} u(x)+\sum_{|\alpha: l|<1} A_{\alpha}(x) D^{[\alpha]} u(x)=f(x),
$$

where $G \subset \mathbb{R}^{n}$ is a region of cylindrical type, $x=\left(x_{1}, x_{2}, \ldots, x_{n}\right), D_{k}^{[i]} u(x)=\left(x_{k}^{\gamma_{k}}(d /\right.$ $d x))^{i} u(x), 0 \leq \gamma_{k}<p-1$, and $A, A_{\alpha}$ are, generally speaking, unbounded operators in $E$.

Under the substitution

$$
y_{k}=\left(1-\gamma_{k}\right)^{-1} x_{k}^{1-\gamma_{k}}
$$

the spaces $L_{p}(G ; E)$ and $W_{p, \gamma}^{[2]}(G ; E(A), E)$ are mapped isomorphically onto the weighted spaces $L_{p, \tilde{\gamma}}(\tilde{G} ; E)$ and $W_{p, \tilde{\gamma}}^{2}(\tilde{G} ; E(A), E)$, respectively, where

$$
\tilde{\gamma}=\prod_{k=1}^{n}\left(1-\gamma_{k}\right)^{\gamma_{k} /\left(1-\gamma_{k}\right)} y^{\gamma_{k} /\left(1-\gamma_{k}\right)} .
$$

Moreover, under the substitution (6.13), the corresponding degenerate boundary value problem reduces to the nondegenerate boundary value problem (6.1).

\section{Nonlocal boundary value problems}

7.1. Quasielliptic equations. The Fredholm property of boundary value problems for elliptic equations in smooth domains was studied in $[1,2,3,23]$. The case of nonsmooth domains was treated in $[17,20,27,36]$, for instance. Let $\Omega_{0}=G \times \Omega$, where $\Omega \subset \mathbb{R}^{m}$, $m \geq 2$, is a bounded domain with an $(m-1)$-dimensional boundary $\partial \Omega$ which locally admits rectification. We consider a nonlocal boundary value problem for a quasielliptic 
differential equation of the form

$$
\begin{aligned}
L u= & \sum_{k=1}^{2}(-1)^{l_{k}} a_{k} D_{k}^{2 l_{k}} u(x, y)+\sum_{|\alpha: 2 l|<1} \sum_{j=1}^{n} a_{\alpha j}(x, y) D_{x}^{\alpha} D_{j} u(x, y) \\
& +\sum_{k, j=1}^{m} a_{k j}(y) D_{k} D_{j} u(x, y)+b(x, y) u(x, y)=f(x, y), \quad(x, y) \in \Omega_{0}, \\
L_{1 j} u= & \sum_{i=0}^{m_{1 j}}\left[\alpha_{1 j i} u_{x_{1}}^{(i)}\left(0, x_{2}, y\right)+\beta_{1 j i} u_{x_{1}}^{(i)}\left(1, x_{2}, y\right)+\sum_{\nu=1}^{N_{1 j}} \delta_{1 j i v} u_{x_{1}}^{(i)}\left(x_{v}, x_{2}, y\right)\right]=0, \quad j=1,2, \ldots, 2 l_{1}, \\
L_{2 j} u= & \sum_{i=0}^{m_{2 j}}\left[\alpha_{2 j i} u_{x_{2}}^{(i)}\left(x_{1}, 0, y\right)+\beta_{2 j i} u_{x_{2}}^{(i)}\left(x_{1}, h, y\right)+\sum_{\nu=1}^{N_{2 j}} \delta_{2 j i v} u_{x_{2}}^{(i)}\left(x_{1}, \Gamma_{v}, y\right)\right]=0, \\
L_{0} u= & \sum_{j=1}^{m} c_{j}\left(y^{\prime}\right) \frac{\partial}{\partial y_{j}} u\left(x, y^{\prime}\right)+c_{0}\left(y^{\prime}\right) u\left(x, y^{\prime}\right)=0, \quad x \in G, \quad y^{\prime} \in \partial \Omega,, \ldots, 2 l_{2},
\end{aligned}
$$

where

$$
x \in G=\left\{\left(x_{1}, x_{2}\right) \in \mathbb{R}^{2}: 0<x_{1}<1,0<x_{2}<h\left(x_{1}\right)\right\}, \quad y=\left(y_{1}, y_{2}, \ldots, y_{m}\right) \in \Omega,
$$

$h$ is a continuous function on [0,1], $0<x_{\nu}<1,0<\Gamma_{\nu}<h\left(x_{1}\right)$, and $a_{k}, \alpha_{k j i}, \beta_{j i}, \delta_{k j i v}$ are complex numbers. We denote $\alpha_{k j m_{k j}}$ by $\alpha_{k j}$ and $\beta_{k j m_{k j}}$ by $\beta_{k j}, k=1,2$. Let $D_{k}^{\alpha_{k}}=\partial^{\alpha_{k}} / \partial x^{\alpha_{k}}$, $D^{\alpha}=D_{1}^{\alpha_{1}} D_{2}^{\alpha_{2}}, D_{j}=-i\left(\partial / \partial y_{j}\right)$. Let $r=\operatorname{ord} L_{0}$ and $\Omega_{0}=G \times \Omega$.

THeOREM 7.1. Suppose that the following conditions are satisfied:

(1) $a_{k j} \in C(\bar{\Omega}), \partial \Omega \in C^{2}, \gamma\left(x_{1}, x_{2}\right)=x_{1}^{\gamma_{1}} x_{2}^{\gamma_{2}}, 0 \leq \gamma_{k}<1-1 / p$;

(2) $\sum_{j=1}^{m} c_{j}\left(y^{\prime}\right) \sigma_{j} \neq 0, y^{\prime} \in \partial \Omega, \sigma \in \mathbb{R}^{m} \backslash\{0\}$ for $r=1$; $c_{0}\left(y^{\prime}\right) \neq 0, y^{\prime} \in \partial G$ for $r=0$;

(3) for $y \in \bar{\Omega}, \sigma \in \mathbb{R}^{m}, \arg \lambda=\pi,|\sigma|+|\lambda| \neq 0, \lambda+\sum_{k, j=1}^{m} a_{k j}(y) \sigma_{k} \sigma_{j} \neq 0$;

(4) $y^{\prime}$ is any point on $\partial \Omega$, the vector $\sigma^{\prime}$ is tangent, and $\sigma$ is a normal vector to $\partial \Omega$ at the point $y^{\prime} \in \partial \Omega$. Consider the boundary value problem

$$
\begin{gathered}
{\left[\lambda+\sum_{k, j=1}^{m} a_{k j}\left(y^{\prime}\right)\left(\sigma_{k}^{\prime}-i \sigma_{j} \frac{d}{d t}\right)\left(\sigma_{k}^{\prime}-i \sigma_{j} \frac{d}{d t}\right)\right] u(\xi)=0, \quad \xi>0, \lambda \leq 0,} \\
\left.\sum_{j=1}^{m} c_{j}\left(y^{\prime}\right)\left(\sigma_{k}^{\prime}-i \sigma_{j} \frac{d}{d t}\right) u(\xi)\right|_{\xi=0}=h \quad \text { for } r=1, \\
u(0)=h \quad \text { for } r=0 .
\end{gathered}
$$

It is required that for $r=1$ problem (7.3), (7.4) (for $r=0$ problem (7.3), (7.5)) has one and only one solution, including all its derivatives, tending to zero as $\xi \rightarrow \infty$ for any $h \in C^{1}$; 
(5) $a_{k}$ are positive numbers and

$$
\eta_{1}, \eta_{2} \neq 0, \quad a_{\alpha, j}, b \in L_{\infty}\left(\Omega_{0}\right) .
$$

Then,

(1) the problem (7.1) for $f \in L_{\mathbf{p}, \gamma}\left(\Omega_{0}\right), \mathbf{p}=\left(p_{1}, p_{2}\right), p_{1}, p_{2} \in(1, \infty)$ has a unique solution that belongs to the space $W_{\mathbf{p}, \gamma}^{(2 l, 2)}\left(\Omega_{0}\right)$, and coercivity, that is,

$$
\|u\|_{W_{\mathbf{p}, \gamma}^{(2,2)}\left(\Omega_{0}\right)} \leq C\left[\|L u\|_{L_{\mathbf{p}, \gamma}\left(\Omega_{0}\right)}+\|u\|_{L_{\mathbf{p}, \gamma}\left(\Omega_{0}\right)}\right]
$$

holds for the solution of the problem (7.1);

(2) the problem (7.1) is Fredholm in $L_{\mathbf{p}, \gamma}\left(\Omega_{0}\right)$.

Proof. Let $E=L_{p_{2}}(\Omega)$ and consider an operator $A$ which is defined by the equalities

$$
\begin{gathered}
D(A)=W_{p_{2}}^{2}\left(\Omega ; L_{0} u=0\right), \quad A u=-\sum_{k, j=1}^{m} a_{k j}(y) D_{k} D_{j} u(y), \\
A_{\alpha}(x) u=\sum_{j=1}^{n} a_{\alpha j}(x, y) D_{j} u(x, y)+b(x, y) u(x, y) .
\end{gathered}
$$

Then, the problem (7.1) can be rewritten in the form

$$
L u=\sum_{k=1}^{2}(-1)^{l_{k}} a_{k} D_{k}^{2 l_{k}} u(x)+\sum_{|\alpha: 2 l|<1} a_{\alpha}(x) D^{\alpha} u(x)+A u(x)=f(x), \quad x \in G, L_{k j} u=0,
$$

where $u(x)=u(x, \cdot)$ and $f(x)=f(x, \cdot)$ are functions with values in the Banach space $E=L_{p_{2}}(\Omega), f_{k}=f_{k}(\cdot)$.

We apply Theorems 5.2 and 5.3 to problem (7.9). By virtue of [21], the space $E=L_{p_{2}}$ satisfies the multiplier condition with respect to $p$ and with respect to the weight function $x^{\gamma}$. By [1], the problem

$$
\lambda u(y)+\sum_{k, j=1}^{m} a_{k j}(y) D_{k} D_{j} u(y)=f(y), \quad y \in G
$$

for $\arg \lambda=\pi,|\lambda| \rightarrow \infty$ and $f \in L_{p}(\Omega)$, has a unique solution $u \in W_{p_{2}}^{2}(\Omega)$ and

$$
|\lambda|\|u\|_{L_{p_{2}}(\Omega)} \leq C\|f\|_{L_{p_{2}}(\Omega)} .
$$

Consequently,

$$
\left\|(A-\lambda I)^{-1}\right\| \leq C|\lambda|^{-1}, \quad \arg \lambda=\pi,|\lambda| \rightarrow \infty .
$$


The operator $A$ is positive in $E=L_{p_{2}}(\Omega)$. It is known (see, e.g., [39]) that for $E=L_{q}(\Omega)$ the definition of $R$-boundedness is reduced to the formula

$$
\left\|\left(\sum_{j=1}^{n}\left|T_{j} u_{j}\right|^{2}\right)^{1 / 2}\right\|_{L_{q}} \leq C\left\|\left(\sum_{j=1}^{n}\left|u_{j}\right|^{2}\right)^{1 / 2}\right\|_{L_{q}} .
$$

Note that by Kahane's inequality (see, e.g., [10]) we can replace $L_{q}$ by $L_{2}$. In Hilbert spaces, all positive operators are $R$-positive. Therefore, using positivity of $A$ and by virtue of the above estimate, we obtain that the operator $A$ is $R$-positive in $L_{p_{2}}$. Moreover, it is known that the embedding $W_{p_{2}}^{2}(G) \subset L_{p_{2}}(G)$ is compact (see, e.g., Triebel [38, Theorem 3.2.5 in Section 3]). Then using interpolation properties of Sobolev spaces [38, Section 4] we obtain that all conditions of Theorems 5.2 and 5.3 are fulfilled. This completes the proof.

7.2. Infinite systems of quasielliptic equations. Consider a BVP

$$
\begin{aligned}
\left(L_{m}+\lambda\right) u= & \sum_{k=1}^{n} a_{k} D_{k}^{l_{k}} u_{m}(x)+\left(b_{m}+\lambda\right) u_{m}(x) \\
& +\sum_{|\alpha: l|<1} \sum_{j=1}^{\infty} b_{\alpha j m}(x) D^{\alpha} u_{m}(x)=f_{m}(x), \\
L_{k v_{k}} u_{m}= & \sum_{i=0}^{q k v_{k}}\left[\alpha_{k v_{k} i} u^{(i)}\left(\Gamma_{k 0}\right)+\beta_{k v_{k} i} u^{(i)}\left(\Gamma_{k T}\right)\right. \\
& \left.+\sum_{j=1}^{N_{k v_{k}}} \delta_{k v_{k} i j} u_{m}^{(i)}\left(\Gamma_{k j d}\right)+\sum_{j=1}^{M_{k v_{k}}} T_{k v_{k} j} u_{m}\left(\Gamma_{k j d 0}\right)\right], \quad m \in \mathbb{N},
\end{aligned}
$$

on $G \subset \mathbb{R}^{n}$, where

$$
\begin{gathered}
G=\left\{x=\left(x_{1}, x_{2}, \ldots, x_{n}\right): 0<x_{k}<T_{k}\right\}, \quad 0 \leq q_{k v_{k}} \leq l_{k}-1, \\
\Gamma_{k 0}=\left(x_{1}, \ldots, x_{k-1}, 0, \ldots, x_{n}\right), \quad \Gamma_{k T}=\left(x_{1}, \ldots, x_{k-1}, T_{k}, \ldots, x_{n}\right), \\
\Gamma_{k j d}=\left(x_{1}, \ldots, x_{k-1}, d_{k j}, \ldots, x_{n}\right), \quad \Gamma_{k j d 0}=\left(x_{1}, \ldots, x_{k-1}, d_{k j 0}, \ldots, x_{n}\right), \\
d_{k j} \in\left(0, T_{k}\right), \quad d_{k j 0} \in\left[0, T_{k}\right], \quad \gamma(x)=\prod_{k=1}^{n} x_{k}^{\gamma_{k}} .
\end{gathered}
$$

Let

$$
\begin{gathered}
b=\left\{b_{m}\right\}, \quad b_{m}>0, \quad u=\left\{u_{m}\right\}, \quad b u=\left\{b_{m} u_{m}\right\}, \quad m \in \mathbb{N}, \\
l_{p}(b)=\left\{u: u \in l_{p},\|u\|_{l_{p}}(b)=\|b u\|_{l_{p}}=\left(\sum_{m=1}^{\infty}\left|b_{m} u_{m}\right|^{p}\right)^{1 / p}<\infty\right\}, \\
1 \leq p<\infty .
\end{gathered}
$$


By $\omega_{k j}, j=1,2, \ldots, l_{k}, k=1,2, \ldots, n$, we will denote the roots of the equations

$$
a_{k} \omega^{l_{k}}+1=0, \quad k=1,2, \ldots, n .
$$

Let $\left[v_{k j i}\right], i, j=1,2, \ldots, l_{k}$ be $l_{k}$-dimensional matrices with determinant $\eta_{k}=\operatorname{det}\left[v_{k j i}\right]$, where (5.5) holds for $0<d_{k}<l_{k}, j=1,2, \ldots, l_{k}, k=1,2, \ldots, n$.

Let $Q$ denote a differential operator in $L_{p, \gamma}\left(G ; l_{p}\right)$ generated by the boundary value problem (7.14), (7.15). Let $B=B\left(L_{p, \gamma}\left(G ; l_{p}\right)\right)$.

Theorem 7.2. Let $\eta_{k} \neq 0,0 \leq \gamma_{k}<1-1 / p, k=1,2, \ldots, n$, and let $b_{m}$ be positive numbers, $b_{\alpha j m} \in L_{\infty}\left(\mathbb{R}^{n}\right)$ such that

$$
\sum_{j=1}^{\infty} \sum_{m=1}^{\infty} b_{\alpha j m}^{p}(x) b_{m}^{-(1-|\alpha: l|) p}<\infty
$$

a.e. for $x \in G$ and $0<\mu<1-|\alpha: l|, 1<p<\infty$. Then,

(1) for all $f(x)=\left\{f_{m}(x)\right\}_{m \in \mathbb{N}} \in L_{p, \gamma}\left(G ; l_{p}\right)$ and for sufficiently large $\lambda>0$, the problem (7.14), (7.15) has a unique solution $u=\left\{u_{m}(x)\right\}_{m \in \mathbb{N}}$ that belongs to the space $W_{p, \gamma}^{l}\left(G, l_{p}(b), l_{p}\right)$, and coercivity, that is,

$$
\begin{gathered}
\sum_{k=1}^{n}\left[\int_{G} \sum_{m=1}^{\infty}\left|D_{k}^{l_{k}} u_{m}(x)\right|^{p} \gamma(x) d x\right]^{1 / p}+\left[\int_{G} \sum_{m=1}^{\infty}\left|b_{m} u_{m}(x)\right|^{p} \gamma(x) d x\right]^{1 / p} \\
\leq C\left[\int_{G} \sum_{m=1}^{\infty}\left|f_{m}(x)\right|^{p} \gamma(x) d x\right]^{1 / p},
\end{gathered}
$$

holds for the solution of the problem (7.14), (7.15);

(2) for sufficiently large $\lambda>0$, there exists a resolvent $\left(Q_{0}+\lambda\right)^{-1}$ of the operator $Q_{0}$, and the following estimate is obtained:

$$
\sum_{k=1}^{n} \sum_{j=0}^{l_{k}}(1+|\lambda|)^{1-j / l_{k}}\left\|D_{k}^{j}\left(Q_{0}+\lambda\right)^{-1}\right\|_{B}+\left\|A\left(Q_{0}+\lambda\right)^{-1}\right\|_{B} \leq M .
$$

Proof. Let $E=l_{p}, A$ and $A_{\alpha}(x)$ be infinite matrices such that

$$
A=\left[b_{m} \delta_{m j}\right], \quad A_{\alpha}(x)=\left[b_{\alpha j m}(x) \delta_{m j}\right], \quad m, j \in \mathbb{N} .
$$

It is easy to see that this operator $A$ is positive in $l_{p}$. Therefore, by Theorem 5.2 we obtain that the problem (7.14), (7.15) for all $f \in L_{p, \gamma}\left(G ; l_{p}\right)$ and for sufficiently large $\lambda>0$ has a unique solution $u(x)$ that belongs to the space $W_{p, \gamma}^{l}\left(G ; l_{p}(b), l_{p}\right)$, and

$$
\sum_{k=1}^{n}\left\|D_{k}^{l_{k}} u\right\|_{L_{p, \gamma}\left(G ; l_{p}\right)}+\|b u\|_{L_{p, \gamma}\left(G ; l_{p}\right)} \leq C\|f\|_{L_{p, \gamma}\left(G ; l_{p}\right)}
$$

From the above estimate we obtain (7.20). The estimate (7.21) is obtained from (6.4). 
Remark 7.3. There are many positive operators in different specific Banach spaces. Therefore, putting instead of $E$ specific Banach spaces and instead of the operator $A$ specific positive differential, pseudodifferential operators, or finite or infinite matrices for instance, on the differential-operator equations (2.31), by Theorems 5.2 and 5.3 we can obtain a different class of coercive and Fredholm boundary value problems for partial differential or pseudodifferential equations or its finite and infinite systems.

Remark 7.4. By virtue of Theorem 5.2, we can obtain estimates of type (5.30) for the boundary value problem (7.1).

\section{References}

[1] S. Agmon, On the eigenfunctions and on the eigenvalues of general elliptic boundary value problems, Comm. Pure Appl. Math. 15 (1962), 119-147.

[2] S. Agmon and L. Nirenberg, Properties of solutions of ordinary differential equations in Banach space, Comm. Pure Appl. Math. 16 (1963), 121-239.

[3] M. S. Agranovich and M. I. Vishik, Elliptic problems with a parameter and parabolic problems of general type, Uspekhi Mat. Nauk 19 (1964), no. 3(117), 53-161.

[4] H. Amann, Linear and Quasilinear Parabolic Problems. Vol. I, Monographs in Mathematics, vol. 89, Birkhäuser Boston, Massachusetts, 1995.

[5] _ Operator-valued Fourier multipliers, vector-valued Besov spaces, and applications, Math. Nachr. 186 (1997), 5-56.

[6] J.-P. Aubin, Abstract boundary-value operators and their adjoints, Rend. Sem. Mat. Univ. Padova 43 (1970), 1-33.

[7] O. V. Besov, V. P. Il'in, and S. M. Nikol'skiĭ, Integral Representations of Functions, and Embedding Theorems, 2nd ed., Fizmatlit Nauka, Moscow, 1996.

[8] J. Bourgain, Some remarks on Banach spaces in which martingale difference sequences are unconditional, Ark. Mat. 21 (1983), no. 2, 163-168.

[9] D. L. Burkholder, A geometric condition that implies the existence of certain singular integrals of Banach-space-valued functions, Conference on Harmonic Analysis in Honor of Antoni Zygmund, Vol. I, II (Chicago, Ill, 1981), Wadsworth Math. Ser., Wadsworth, California, 1983, pp. 270-286.

[10] J. Diestel, H. Jarchow, and A. Tonge, Absolutely Summing Operators, Cambridge Studies in Advanced Mathematics, vol. 43, Cambridge University Press, Cambridge, 1995.

[11] G. Dore and A. Venni, On the closedness of the sum of two closed operators, Math. Z. 196 (1987), no. 2, 189-201.

[12] G. Dore and S. Yakubov, Semigroup estimates and noncoercive boundary value problems, Semigroup Forum 60 (2000), no. 1, 93-121.

[13] N. Dunford and J. T. Schwartz, Linear Operators. Part II. Spectral Theory. Self adjoint Operators in Hilbert Space, John Wiley \& Sons, New York, 1988, with the assistance of William G. Bade and Robert G. Bartle; reprint of the 1963 original.

[14] A. Favini, Su un problema ai limiti per certe equazioni astratte del secondo ordine, Rend. Sem. Mat. Univ. Padova 53 (1975), 211-230 (Italian).

[15] V. I. Gorbachuk and M. L. Gorbachuk, Boundary Value Problems for Operator Differential Equations, Mathematics and Its Applications (Soviet Series), vol. 48, Kluwer Academic Publishers, Dordrecht, 1991.

[16] M. Hieber and J. Prüss, Heat kernels and maximal $L^{p}-L^{q}$ estimates for parabolic evolution equations, Comm. Partial Differential Equations 22 (1997), no. 9-10, 1647-1669.

[17] D. S. Jerison and C. E. Kenig, The Dirichlet problem in nonsmooth domains, Ann. of Math. (2) 113 (1981), no. 2, 367-382. 
[18] H. I. Karakas, V. B. Shakhmurov, and S. Yakubov, Degenerate elliptic boundary value problems, Appl. Anal. 60 (1996), no. 1-2, 155-174.

[19] T. Kato, Perturbation Theory for Linear Operators, Classics in Mathematics, Springer, Berlin, 1995.

[20] V. A. Kondrat'ev and O. A. Oleŭnik, Boundary value problems for partial differential equations in nonsmooth domains, Russian Math. Surveys 38 (1983), no. 2, 1-86.

[21] P. Krée, Sur les multiplicateurs dans $\mathscr{F}^{L^{p}}$ avec poids, Ann. Inst. Fourier (Grenoble) 16 (1966), 91-121 (French).

[22] S. G. Kreĭn, Linear Differential Equations in Banach Space, American Mathematical Society, Rhode Island, 1971.

[23] J.-L. Lions and E. Magenes, Problèmes aux limites non homogénes. VI, J. Analyse Math. 11 (1963), 165-188 (French).

[24] J.-L. Lions and J. Peetre, Sur une classe d'espaces d'interpolation, Inst. Hautes Études Sci. Publ. Math. 19 (1964), 5-68 (French).

[25] P. I. Lizorkin, $\left(L_{p}, L_{q}\right)$-multipliers of Fourier integrals, Dokl. Akad. Nauk SSSR 152 (1963), 808811.

[26] T. R. McConnell, On Fourier multiplier transformations of Banach-valued functions, Trans. Amer. Math. Soc. 285 (1984), no. 2, 739-757.

[27] S. A. Nazarov and B. A. Plamenevsky, Elliptic Problems in Domains with Piecewise Smooth Boundaries, de Gruyter Expositions in Mathematics, vol. 13, Walter de Gruyter, Berlin, 1994.

[28] G. Pisier, Les inégalités de Khintchine-Kahane, d’après C. Borell, Séminaire sur la Géométrie des Espaces de Banach (1977-1978), École Polytech., Palaiseau, 1978, pp. 1-14.

[29] V. B. Shakhmurov, The compactness of an imbedding into nonisotropic spaces of vector-valued functions and applications, Dokl. Akad. Nauk SSSR 241 (1978), no. 6, 1285-1287.

[30] - Theorems on imbedding compactness in weighted anisotropic spaces and their applications, Dokl. Akad. Nauk SSSR 291 (1986), no. 6, 1305-1309.

[31] _ Embedding theorems in abstract spaces and their applications to degenerate differentialoperator equations, Ph.D. thesis, Steklov Mat. Inst., Moscow, 1987.

[32] - Theorems on the embedding of abstract function spaces and their applications, Mat. Sb. (N.S.) 134(176) (1987), no. 2, 260-273.

[33] Embedding theorems and their applications to degenerate equations, Differentsial nye Uravneniya 24 (1988), no. 4, 672-682.

[34] Embedding theorems in Banach-valued Sobolev-Liouville spaces and their applications, Istanbul University-Journal of Electrical \& Electronics Engineering 2 (2002), no. 2, 575591.

[35] A. Y. Shklyar, Complete Second Order Linear Differential Equations in Hilbert Spaces, Operator Theory: Advances and Applications, vol. 92, Birkhäuser, Basel, 1997.

[36] A. L. Skubachevskii, Elliptic Functional-Differential Equations and Applications, Operator Theory: Advances and Applications, vol. 91, Birkhäuser, Basel, 1997.

[37] P. E. Sobolevskiĭ, Coerciveness inequalities for abstract parabolic equations, Dokl. Akad. Nauk SSSR 157 (1964), 52-55.

[38] H. Triebel, Interpolation Theory, Function Spaces, Differential Operators, North-Holland Mathematical Library, vol. 18, North-Holland Publishing, Amsterdam, 1978.

[39] L. Weis, Operator-valued Fourier multiplier theorems and maximal $L_{p}$-regularity, Math. Ann. 319 (2001), no. 4, 735-758.

[40] S. Yakubov, Completeness of Root Functions of Regular Differential Operators, Pitman Monographs and Surveys in Pure and Applied Mathematics, vol. 71, Longman Scientific \& Technical, Harlow, 1994. 
42 Maximal regular BVPs in Banach-valued weighted space

[41] A nonlocal boundary value problem for elliptic differential-operator equations and applications, Integral Equations Operator Theory 35 (1999), no. 4, 485-506.

[42] S. Yakubov and Y. Yakubov, Differential-Operator Equations. Ordinary and Partial Differential Equations, Chapman \& Hall/CRC Monographs and Surveys in Pure and Applied Mathematics, vol. 103, Chapman \& Hall/CRC, Florida, 2000.

Ravi P. Agarwal: Department of Mathematical Sciences, Florida Institute of Technology, Melbourne, FL 32901-6975, USA

E-mail address: agarwal@fit.edu

Martin Bohner: Department of Mathematics, University of Missouri-Rolla, Rolla, MO 654090020, USA

E-mail address: bohner@umr.edu

Veli B. Shakhmurov: Department of Electrical-Electronics Engineering, Faculty of Engineering, Istanbul University, Avcilar, 34850 Istanbul, Turkey

E-mail address: sahmurov@istanbul.edu.tr 